\title{
Experimental investigations on the Common Research Model at ONERA-S2MA
}

\author{
Aurelia CARTIERI ${ }^{1}$ \\ ONERA, the French Aerospace Lab, 73500 Modane, France
}

\begin{abstract}
This paper aims at presenting some of the experimental results obtained with the NASA-Boeing Common Research Model at ONERA. The model used in the present study is the ONERA S2MA Large Reference Model (1/50) which has the same geometry as the CRM considered in the latest AIAA Drag Prediction Workshops. Experimental data have been collected from the ONERA-S2MA wind tunnel at Mach numbers between 0.30 and 0.94 and at chord Reynolds number of 1.8, 2.1, 2.5, 3 and 3.6 million for the WBVH configuration (wing-body with horizontal and vertical tails). Force and moment, surface pressure, infrared measurements and model deformation measurements have been performed. Reynolds number and aeroelastic effects have been assessed. Some comparisons with test data from the ONERA S1MA Large Reference Model (1/16.835) are proposed.
\end{abstract}

\section{Nomenclature}

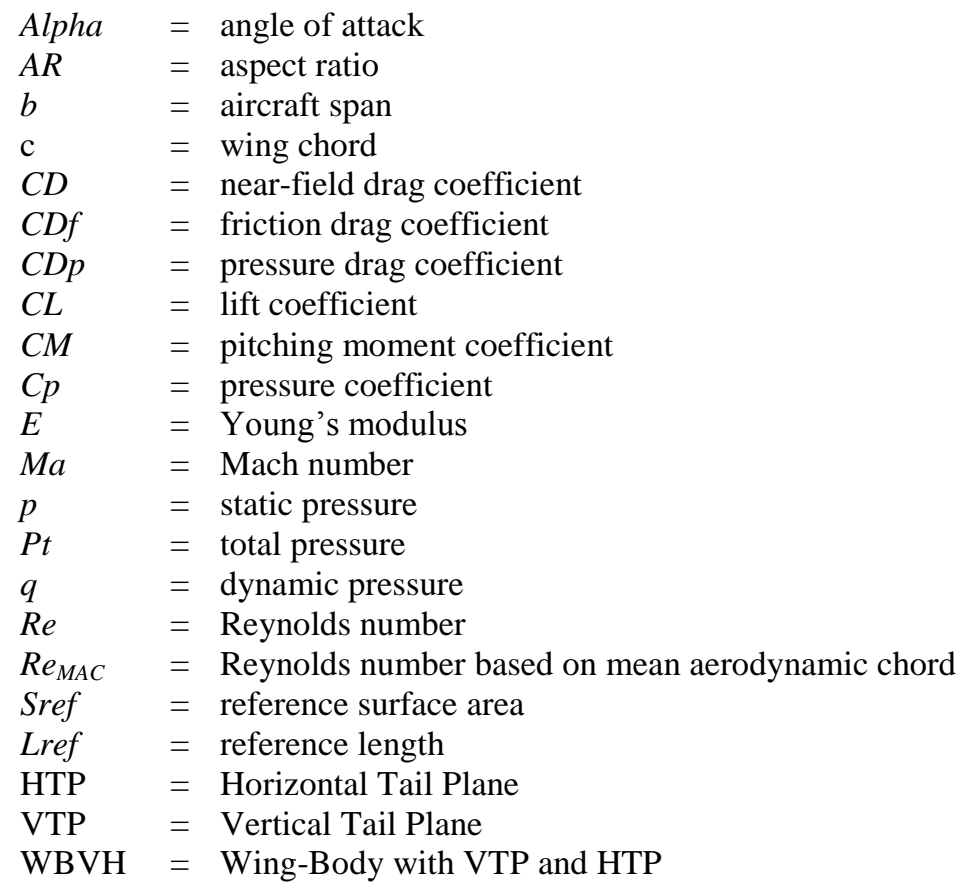

\section{Introduction}

Trio HE Common Research Model (CRM) developed by NASA and Boeing [1,2] serves as a reference for providing wind tunnel data aimed at the validation of codes dedicated to aircraft performance prediction. This model has been designed and built as part of the AIAA Drag Prediction Workshop (DPW) series [3]. For many years now, ONERA has participated to these fruitful workshops [4,5,6,7,8]. In 2014, ONERA has built its first own CRM model; it is called the S1MA Large Reference Model (LRM-S1MA). More recently, ONERA has built its second version of the model; it is called the S2MA Large Reference Model (LRM-S2MA). They both have been designed to have the same geometry as the NASA model when submitted to equivalent constraints.

The ONERA models are used to verify the complete measurement chain of the S1MA and S2MA facilities $[9,10]$. These verifications relate to the wind tunnel structure itself (for instance after repairs and modifications),

\footnotetext{
${ }^{1}$ Engineer, Wind Tunnel Division, aurelia.cartieri@onera.fr AIAA Member.
} 
quality of the airstream, and checkouts of data repeatability over time. Moreover, in the meantime, this model is also used for technical development of new measurement techniques and devices as well as a reference for Computational Fluid Dynamics (CFD) validation.

In May 2019, the first test campaign of the LRM-S2MA took place in S2MA. The main purpose of this test was to acquire a large reference database on this model. Measurements such as force and moment quantification (balance), static skin pressure probing, infrared measurements and model deformation measurements have been performed for large ranges of Mach numbers (0.30 to 0.94) and lift levels. For the moment, only one configuration has been tested: Wing-Body with both HTP and VTP (WBVH). The VTP of LRM has been designed by ONERA; this geometry has been shared with the community and is presented in [11].

In order to highlight the results of these results, the paper will be organized as follows: first, the S2MA wind tunnel and the LRM-S2MA model will be described as well as the test campaign of 2019. Then, experimental results will be presented such as model deformation measurements and Reynolds number/aeroelastic effects. Finally, comparisons will be carried out on the pressure distribution between S1MA and S2MA.

\section{Wind Tunnel Tests}

\section{A. Facility Description}

S2MA is a continuous pressurized wind tunnel operating in the sub/transonic/supersonic regime. It was put into service in 1961 and is driven by a 16-stage axial compressor, driven by four Pelton turbines, the power of which is $57 \mathrm{MW}$. It consists of two interchangeable rectangular test sections installed in a sealed enclosure. In this study, only the transonic test section has been used $(1.75 \mathrm{~m}$ width $\mathrm{x} 1.77 \mathrm{~m}$ height). The total length of the aerodynamic circuit is about $100 \mathrm{~m}$ (see Fig. 1). This test section has porous walls to limit the wall interference and avoid blockage. For a Mach number around 0.85, the Reynolds number per meter can go from approximately 3 to 26 million by varying the dynamic pressure (see Fig. 2).

The temperature is controlled by a water exchanger in the aerodynamic circuit just downstream of the compressor.

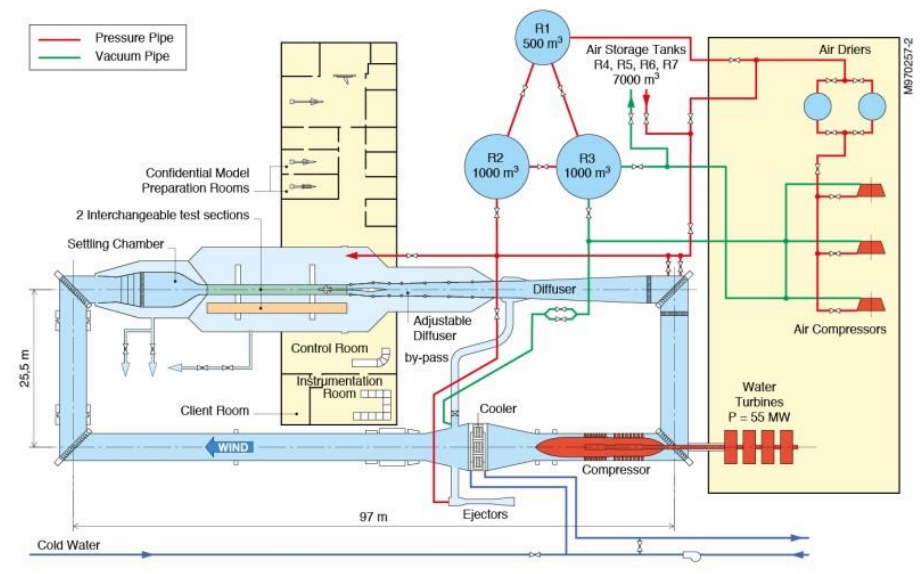

Fig. 1 S2MA air circuit.

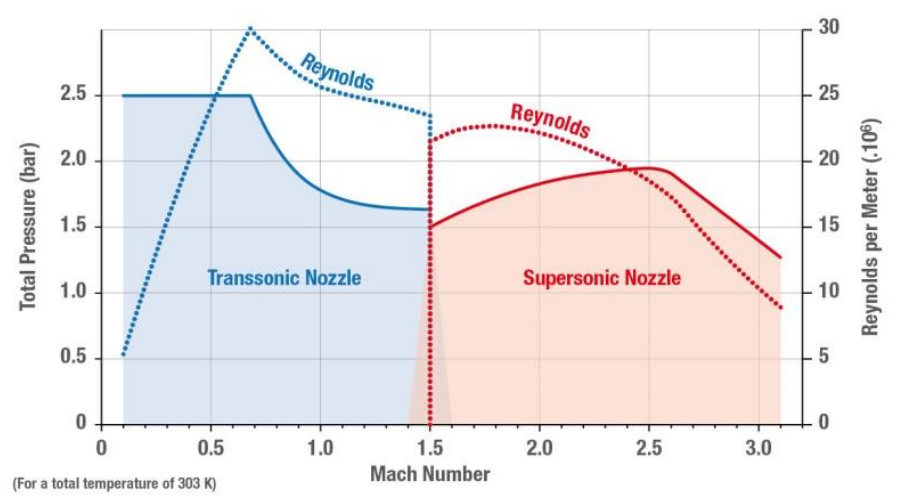

Fig. 2 S2MA operating range. 


\section{B. Model description}

The model used in the current investigation is the ONERA Large Reference Model (LRM-S2MA) which has the same geometry as the CRM. It was scaled to $74 \%$ of the NASA model, so the final scale is $1 / 50$. This configuration consists of a contemporary supercritical transonic wing and a fuselage that are representative of a wide-body commercial transport aircraft. The vertical tail geometry was designed by the ONERA Civil Aircraft Unit of the Applied Aerodynamics Department in cooperation with the Wind Tunnel Division.

The CRM is designed for a cruise Mach number of 0.85 and a corresponding lift coefficient of $C L=0.5$. It exhibits the following characteristics: conventional low wing configuration and high aspect ratio $(A R=9.0)$. The $\mathrm{S} 2$ model is defined by mean aerodynamic chord $L r e f=0.1401 \mathrm{~m}$, reference surface area $S r e f=0.1535 \mathrm{~m}^{2}$ and a span $b=1.1753 \mathrm{~m}$.

The ONERA LRM-S2MA model was designed so that it has the same shape at cruise point (for the highest dynamic pressure in S2MA) as the ONERA-S1MA tested in S1MA wind tunnel and the CRM model tested in the ETW Wind Tunnel. The main dimensions of the model are given in Fig. 3.

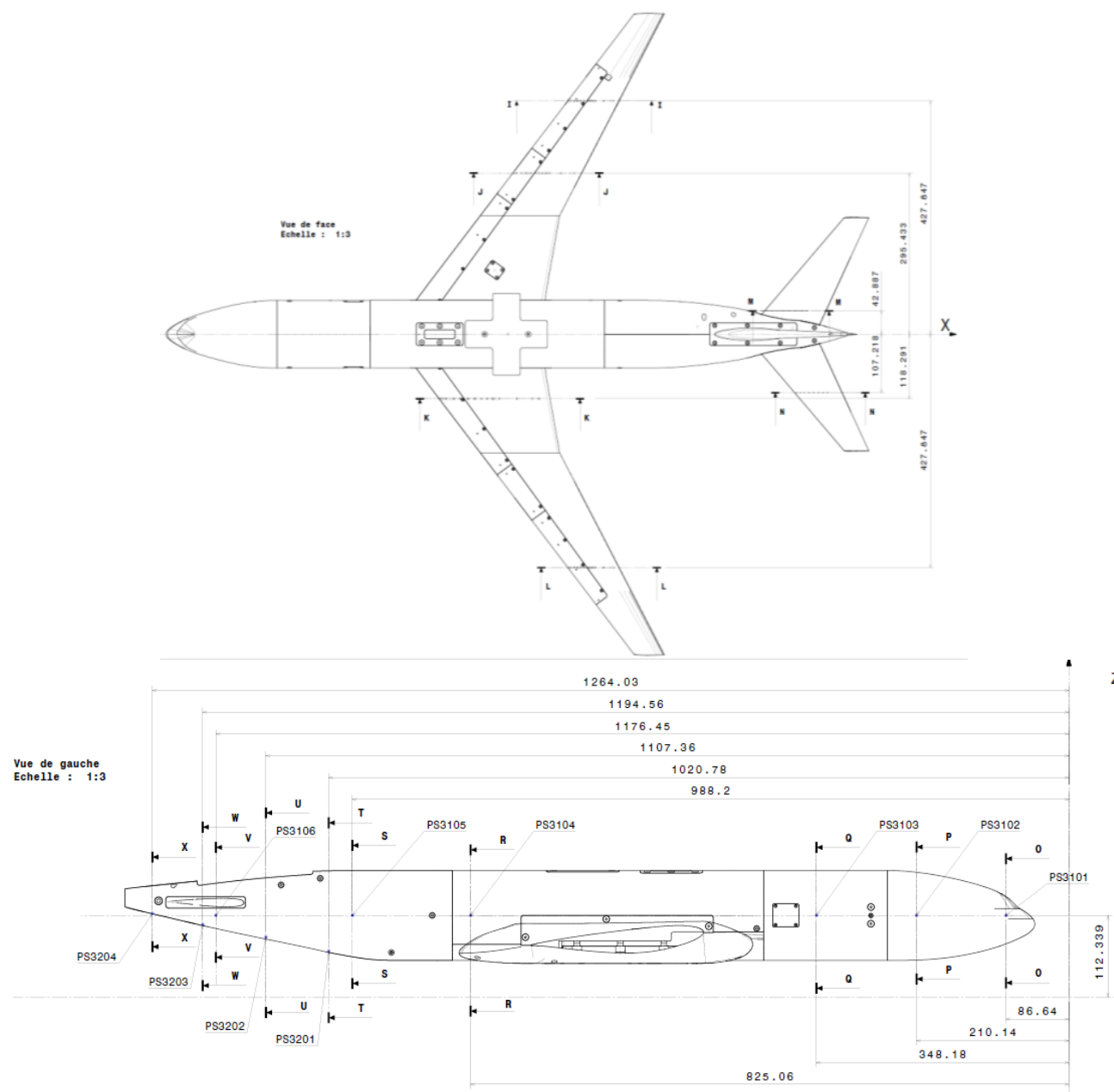

Fig. 3 Main dimensions of the LRM-S2MA model.

Pressure distributions are measured on both the left and right wings using 64 pressure orifices located in four spanwise wing stations : two on the right wing $(\eta=0.502$ and 0.727$)$ and two on the left wing $(\eta=0.201$ and 0.727). There were also two sections on the HTP $(\eta=0.2$ and 0.5$)$. The fuselage was also equipped as shown in Fig. 3.

All pressure measurements were made using Electronically Scanned Pressure (ESP) modules installed inside the forward portion of the fuselage. The model is mounted in the wind tunnel using a $\mathrm{Z}$ sting setup as shown in Fig. 4. 


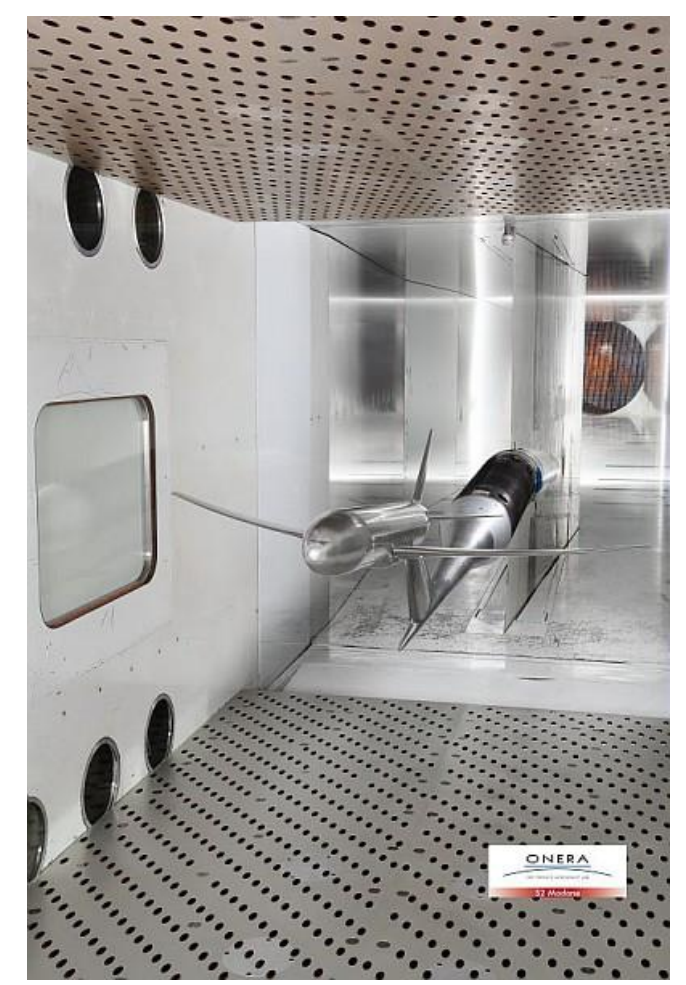

Fig. 4 The LRM-S2MA model in S2MA wind tunnel.

\section{Test conditions and measurements}

The tests were carried out in a Mach number range going from $M a=0.30$ up to $M a=0.94$. Tests were conducted at chord Reynolds number of 1.8, 2.1, 2.5, 3 and 3.6 million. The 2.1, 2.5 and 3 million Reynolds number data were performed for $M a=0.85$ only. The incidence range was from $-3.0^{\circ}$ to $+6.0^{\circ}$. The incidence of the model was measured by means of two goniometers connected to the weighed balance adapter. It was corrected for wall and sting effects (see below) and for the wind tunnel up-wash that was determined during the campaign. The loads of the model were measured with a six-component balance. The wing deformation measurements were performed with two high resolution cameras located in the ceiling of the test section [12] behind a window. The bending and twist deformations of the right wing were derived from the comparison of the 3D target positions between wind-on and wind-off conditions.

The transition of the boundary layer on the different parts of the model was forced by means of CADCUT strips. Trips measuring $1.3 \mathrm{~mm}$ in diameter and spaced $2.4 \mathrm{~mm}$ apart were used for the entire test. The trips dost were installed at $10 \%$ chord on the wings, the HTP and the VTP. The trip dots were $0.089 \mathrm{~mm}$ high on the inner wing and $0.078 \mathrm{~mm}$ high elsewhere. On the fuselage, the trips were applied at $18 \mathrm{~mm}$ from the nose and measured $0.078 \mathrm{~mm}$. Some infrared measurements were performed at the beginning of the test campaign in order to see the natural laminar transition and to check the effectiveness of the boundary layer tripping.

\section{Corrections Methods}

The aerodynamic interferences are taken into account thanks to a correction process composed of several contributions:

1) The empty test section correction: it is a Mach number correction that results from a test section tunnel calibration;

2) The buoyancy correction: it is the effect of the empty wind tunnel Mach number gradient on drag (which is proportional to the product of the gradient and the effective volume of the body);

3) The wall effect correction: these corrections rely on the potential flow theory [13]. Under the assumption that the flow in the tunnel is irrotational outside the boundary layers and wakes, it can be described by a velocity potential $U_{0} x+\varphi$. Assuming now that the velocity perturbations $\partial x \varphi, \partial y \varphi$ and $\partial z \varphi$ are small with regard to $U_{\infty}$, one comes to the well-known linearized potential equation:

$$
\left(1-M a_{\infty}^{2}\right) \partial_{x}^{2} \varphi+\partial_{y}^{2} \varphi+\partial_{z}^{2} \varphi=0
$$

The porous walls are modelled with a velocity through the perforated wall proportional to the pressure drop through the wall. 
This last assumption is less and less valid as the upstream Mach number $M a_{\infty}$ values approach $M a=$ 1.00 and as typical transonic phenomena occur on the model, with large fluid accelerations up to supersonic regime.

This equation and the corresponding boundary conditions can be solved through a distribution of singularities on the model and support. The intensity of each singularity is based on the cross section areas, the lift and the drag.

Once the proper singularities have been set up, the linearity of Eq. (1) allows the potential $\varphi$ to be broken down into a field $\varphi \mathrm{m}$ generated by the model and a field $\varphi s$ generated by the support. Hence $\nabla \varphi s=(u s, v s, w s)$ is the field of velocity distortion generated by the support.

Once the velocity field $\nabla \varphi s$ is known, one can easily determine a field of Mach number distortion:

$$
\delta M a=M a_{\infty}\left(1+\frac{\gamma-1}{2} M a_{\infty}^{2}\right) \frac{u_{s}}{U_{\infty}}(2)
$$

and a field of angle of attack distortion (upwash):

$$
\delta \text { Alpha }=\frac{w_{s}}{U_{\infty}}(\mathbf{3})
$$

These fields are then averaged in space over areas of aerodynamic significance.

The Mach number correction $\Delta M a$ is taken as the value of $\delta M a$ at $1 / 4$ of mean aerodynamic chord. The alpha correction is computed from a slightly more elaborated process: it is chord-averaged along the wing span, at $3 / 4$ of local chord, this correction enabling the lift correction to be zero (theory of Pistolesi, [14]).

Second order corrections on drag (buoyancy correction due to velocity distortion) and pitching moment (mainly due to the HTP lift gradient to alpha) are then calculated;

4) The sting corrections: these corrections are calculated thanks to RANS computations [15]. First order corrections are determined thanks to a pairing process. With and without support simulations are considered as paired when the flow fields around the wing are similar. The criterion of similarity is the RMS of pressure coefficient distortion on the wing. The corrections to forces and moments are deduced from the differences between the integrated forces over the model with and without support.

5) The sting cavity pressure correction: this correction results from the presence of a pressure coefficient (not zero) inside the rear fuselage which is "open" to enable sting entry. It consists in replacing, on the cavity surface, the mean measured cavity pressure by the reference pressure.

\section{Results}

\section{A. Data repeatability}

When data is obtained in any experimental investigation it is important to make an assessment of data repeatability.

\section{Force and moment}

"Short term repeatability" is the comparison of polars performed within the same run or within successive runs for a given configuration. Within each series of runs, two polars were obtained at $M a=0.85$. Fig. 5 presents the superposition of all the short term repeats performed at $M a=0.85$ during the test campaign. Lift is presented versus delta drag, angle of attack, and pitching moment. The deltas represent the difference between the coefficient value measured and the average value of the coefficient at fixed $C L$. This short term repeatability is good (repeatability better than $\pm 0.02^{\circ}, \pm 1$ d.c. and $\pm 0.001 \mathrm{CM}$ ) and is in compliance with the expected standards for a full span model campaign performed over a large test domain. 

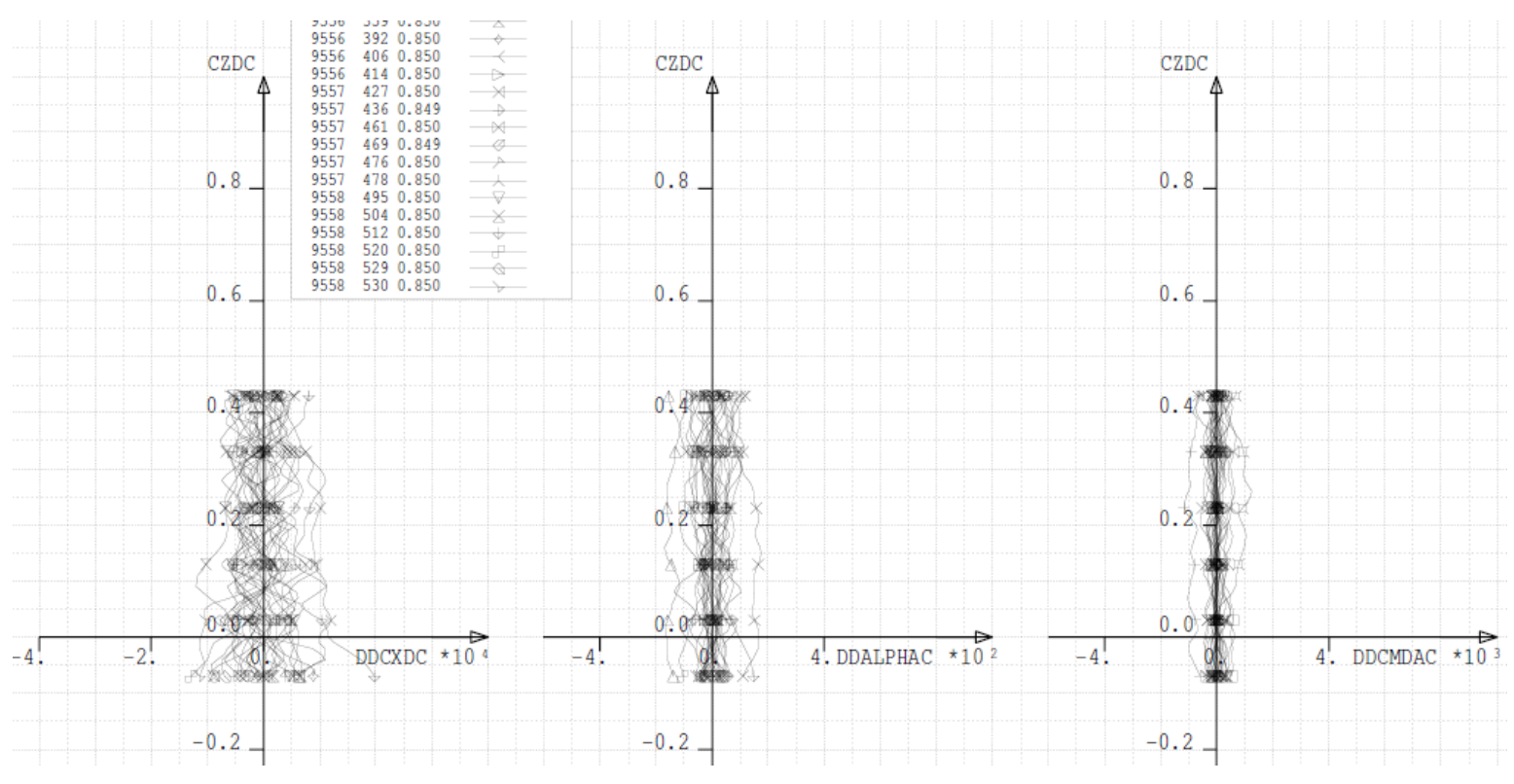

Fig. 5 Short term repeatability for WBVH at $M a=0.85$.

2. Pressure

The pressure distributions obtained on the WBVH configuration at $M a=0.85$ and $C L=0.5$ for two test points are shown in Fig. 6 for the wing and Fig. 7 for the HTP.

The repeatability of the pressure distribution is good.

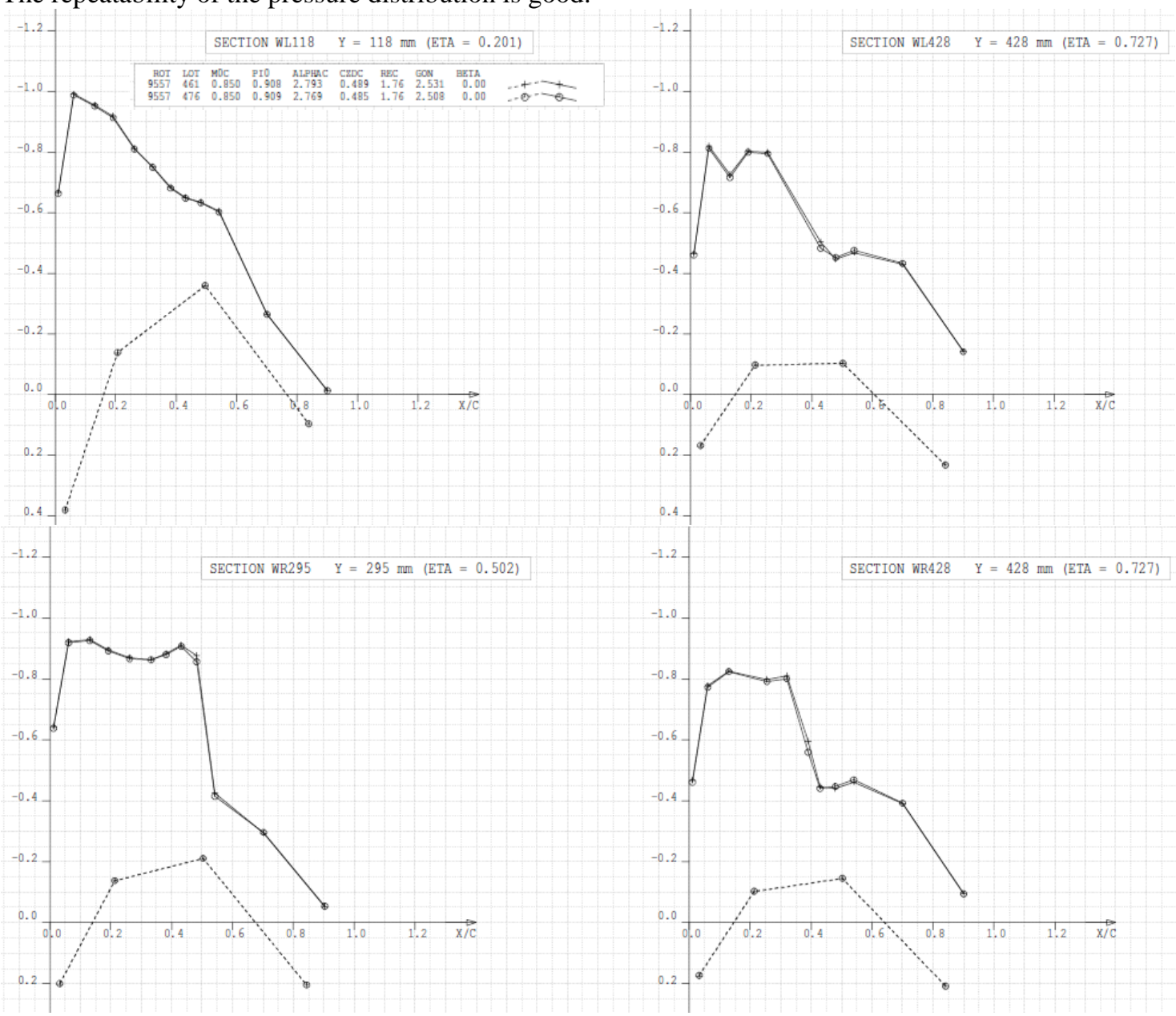

Fig. 6 Repeatability of the pressure distribution on the wing for $\mathrm{WBVH}$ at $M a=0.85$ and $C L=0.5$. 


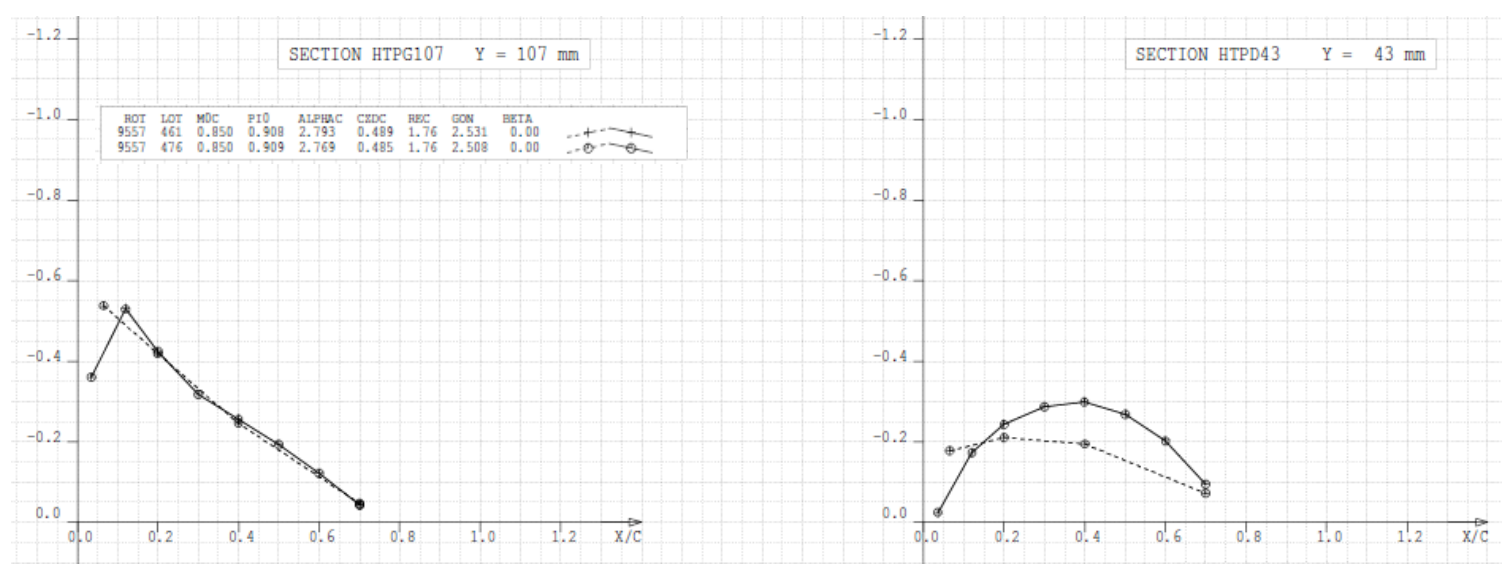

Fig. 7 Repeatability of the pressure distribution on the HTP for WBVH at $M a=0.85$ and $C L=0.5$.

\section{B. Infrared measurements}

Infrared thermography (IRT) was used in order to visualize the boundary layer transition. The status change of the boundary layer from laminar to turbulent can be seen on the flux level and the turbulence level. Infrared thermography is sensitive to the heat flux increase, which allows us to visualize the transition position.

For that purpose, the model was painted with an insulating paint. This paint exhibits a high emissivity and its insulating properties enhance the contrast of the image.

Some visualizations have been carried out on several Mach numbers and lift coefficients, in natural laminar conditions (without any transition trips applied on the wing) and with the transition tripping.

3. Natural laminar

The transition visualization images for two lift coefficient and two total pressure (so two Reynolds number) at $M a=0.85$ can be seen in Fig. 8 on the upper side of the wing and in Fig. 9 on the lower side of the wing.

The dark regions on the image are laminar and the light regions are turbulent. Several features are noted on the images:

- The position of the pressure taps is visible on the images (see green lines on the upper left image of Fig. 8). In fact, the pressure taps were covered during the painting. This isn't usually the case when IRT visualizations are performed but it was done for the present test campaign. These regions don't have any painting, so the emissivity is very low;

- At specific location, some cadcuts were applied in order to trigger the transition (see the red dots on the upper left image of Fig. 8 and Fig. 9). The blue dots are the location of small surface imperfections, causing bypass transition. It is clearly visible that those turbulent wedges are more frequent at high Reynolds numbers (no wedges seen on the right figures at a Reynolds number of 1.7 million) as the boundary layer is thinner.

For the two angle of attack tested (see top and bottom figures on the left), it can be seen that the turbulent wedges occur at the same position suggesting that the imperfections are probably due to the painting itself. The transition position does occur a bit downstream on the upper side at high lift (see Fig. 8), the shock position being located downstream on the outer wing.

The Reynolds number effect on the laminar flow extent is clearly seen on Fig. 9. This shows that the transition location is mainly shock limited on the upper surface, whereas the lower surface exhibits a transition location moving forward as the Reynolds number is increased. 

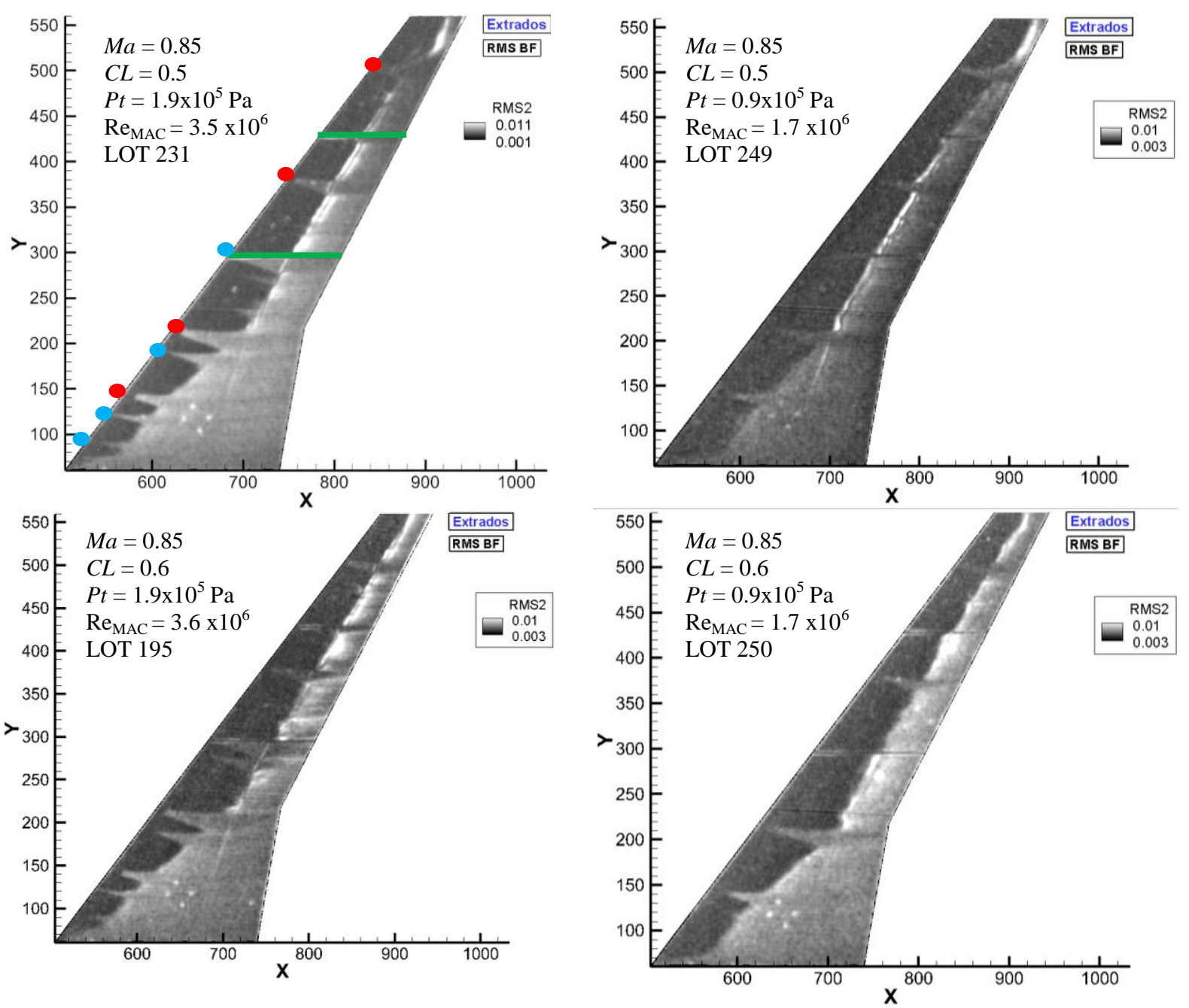

Fig. 8 IRT images on the upper side of the wing at $M a=0.85$. 

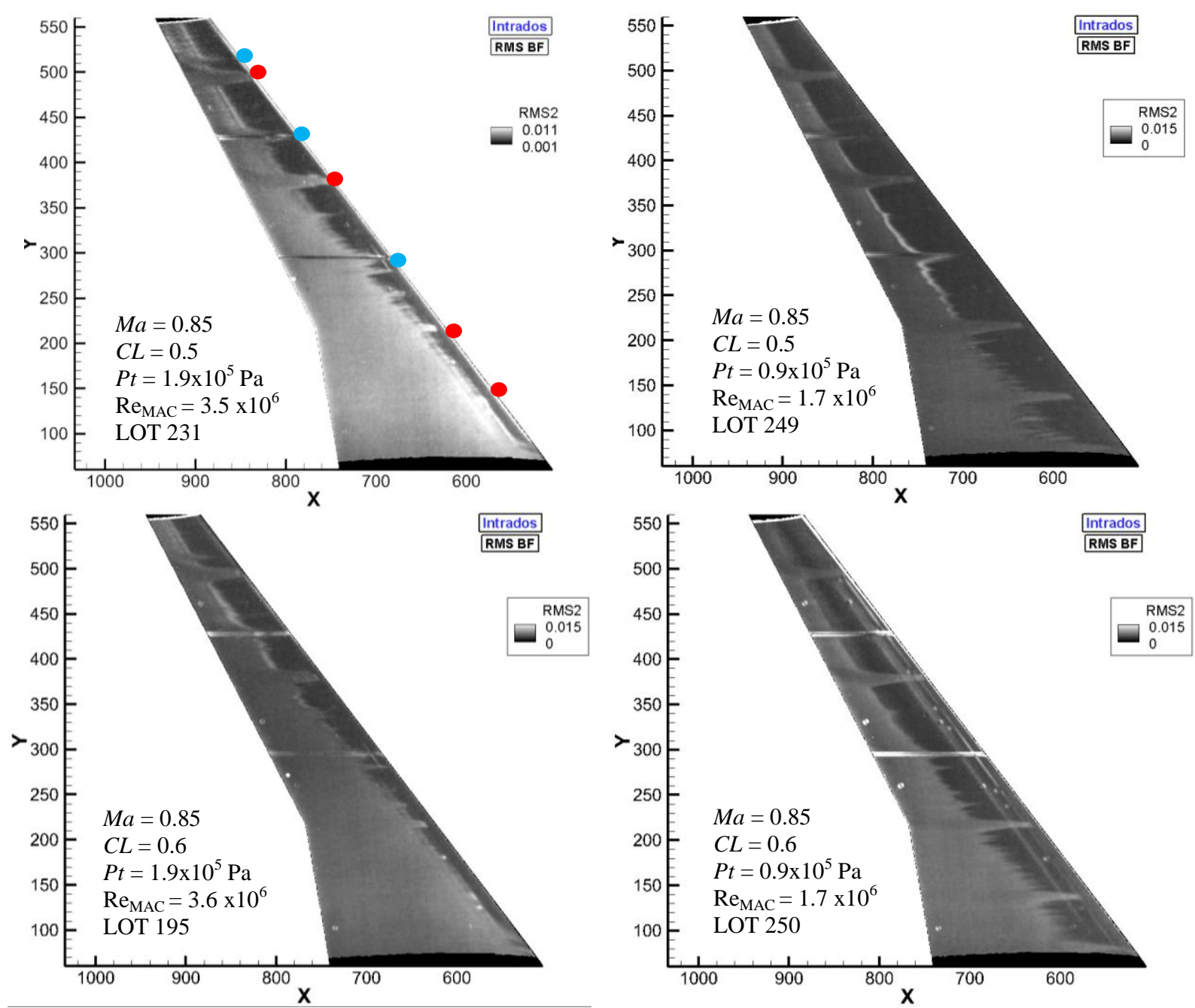

Fig. 9 IRT images on the lower side of the wing at $M a=0.85$.

\section{Imposed transition}

The effectiveness of the boundary layer tripping was then checked.

The transition visualization images for $C L=0.6$ and $M a=0.85$ can be seen in Fig. 10. Images at high Reynolds number are not shown, as some difficulties occurred having a difference of temperature between the model and the wind tunnel flow. However, the effectiveness of the transition is critical at low Reynolds number; the boundary layer thickness being higher.

Some regions didn't have any triggering devices (see the red dots), so the boundary layer transition should be triggered at the same position as the natural laminar images.

It can be seen that:

- On the lower side (right image of Fig. 10), the triggering device is efficient on the whole span. The region without any devices appear in dark;

- On the upper side (left image of Fig. 10), turbulent wakes behind the cadcuts are visible (see bottom image). Generally, a turbulent wedge generated by roughness element would spread in the laminar region with an angle (as it was seen on Fig. 8). Here, we do see that the proximity of each cadcut is sufficient to trigger the boundary layer. The shock itself is also clearly visible. 


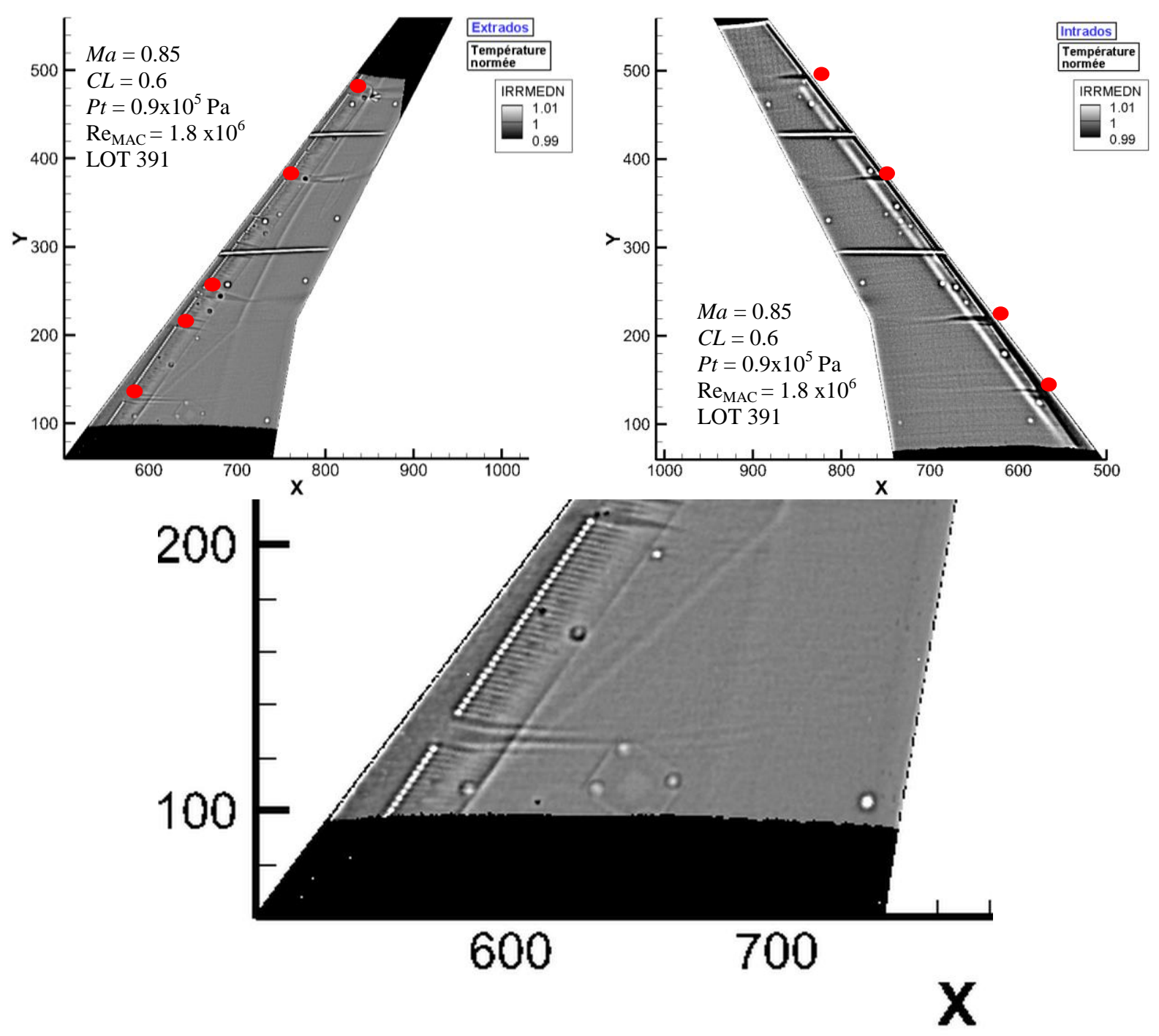

Fig. 10 IRT images on the wing at $M a=0.85$ with triggering device. 


\section{Model Deformation Measurements}

The as-built shape of the two models (LRM-S1MA and LRM-S2MA) is shown in Fig. 11.

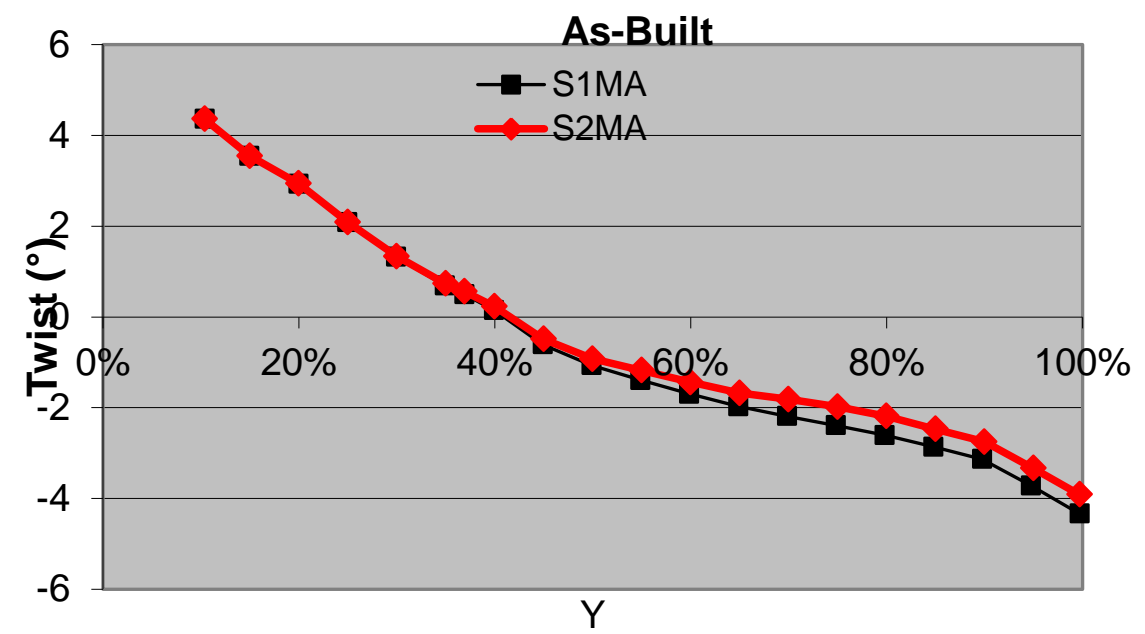

Fig. 11 Model Deformation of LRM-S2MA and LRM-S1MA wings without wind.

During the wind tunnel test, some model deformation measurements were made on the wing. The method used is based on marker detection and stereovision. Some markers are put on the model surface (the wing lower surface), which is imaged by two cameras.

The twist measured at Ma $=0.85$ on LRM-S2MA for the 10 positions on the wing span are showed Fig. 12 for two different lift coefficient.

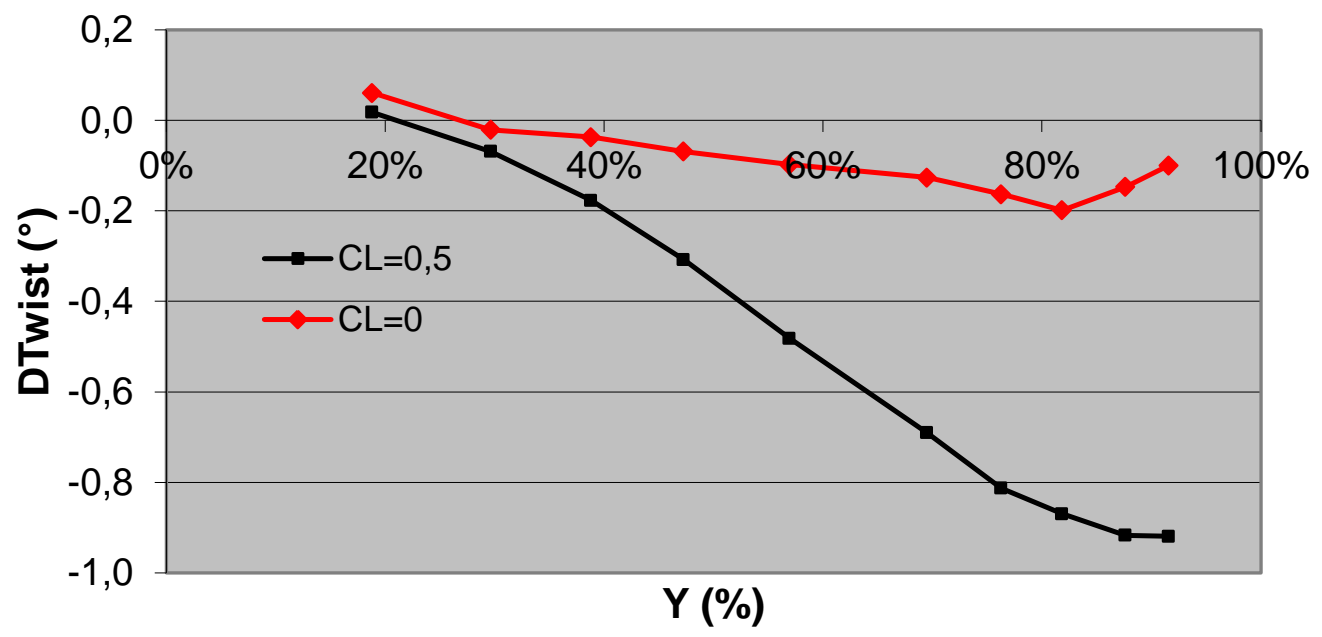

Fig. 12 Model Deformation of LRM-S2MA model at $M a=0.85$ and $\operatorname{Re}_{M A C}=3.6 \times 10^{6}$.

The twist comparison between S1MA and S2MA is made in Fig. 13 at $M a=0.85$ (and for two different lift coefficients). The shape is the same between S1MA and S2MA at cruise point, but differences are clearly seen at low lift. 

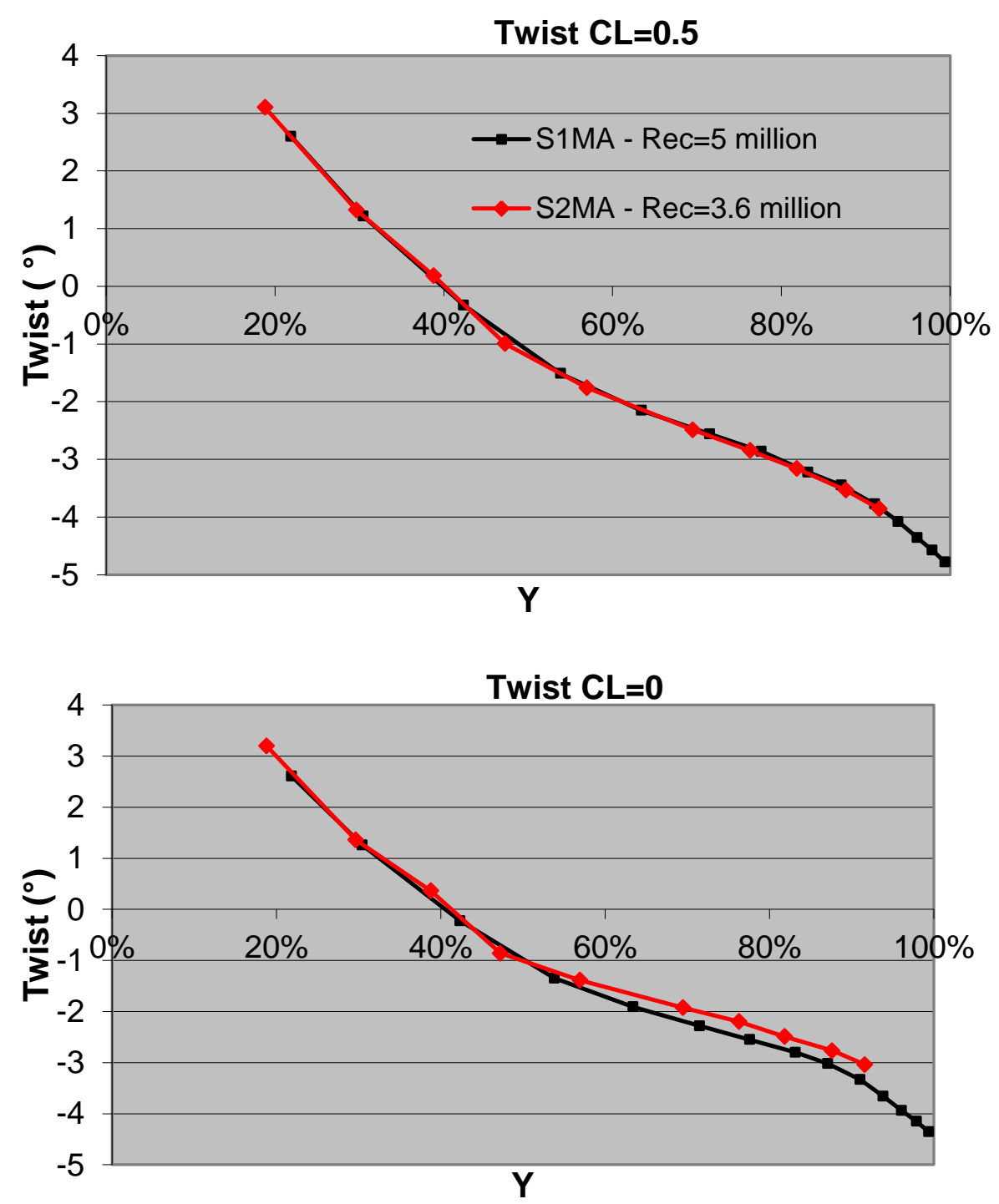

Fig. 13 Model Deformation of LRM-S2MA and LRM-S1MA models at $M a=0.85$.

\section{Reynolds number and aeroelastic effects on model deformation}

Aeroelastic effects were performed at $M a=0.85$. These effects were carried out by changing the total pressure and keeping the total temperature constant. As a consequence, the Reynolds number is also modified. The shape of the wing depends on the non-dimensional ratio $\mathrm{q} / \mathrm{E}$, so the pure Reynolds number change is not causing the model to deform. In Table 1 are presented the test conditions.

Table 1 Test conditions of aeroelastic effects (with Reynolds number change).

\begin{tabular}{|c|c|c|c|}
\hline $\mathbf{n}^{\circ}$ & $\mathbf{M a}$ & $\mathbf{P t}(\mathbf{P a})$ & $\mathbf{R e}_{\text {MAC }}$ (million) \\
\hline $\mathbf{4 7 8}$ & $\mathbf{0 . 8 5}$ & 190000 & 3.6 \\
\hline $\mathbf{4 7 9}$ & $\mathbf{0 . 8 5}$ & 160000 & 3.0 \\
\hline $\mathbf{4 8 0}$ & $\mathbf{0 . 8 5}$ & 131000 & 2.5 \\
\hline $\mathbf{4 8 1}$ & $\mathbf{0 . 8 5}$ & 110000 & 2.1 \\
\hline $\mathbf{4 7 6}$ & $\mathbf{0 . 8 5}$ & 91000 & 1.8 \\
\hline
\end{tabular}

When total pressure is increased keeping all other parameters constant, the load on the model increases. As a consequence, the wing bending increases. This bending translates into aerodynamic twist causing the outer wing section to pitch nose down. This is seen in Fig. 14 which shows the wing twist deformation for two conditions. 


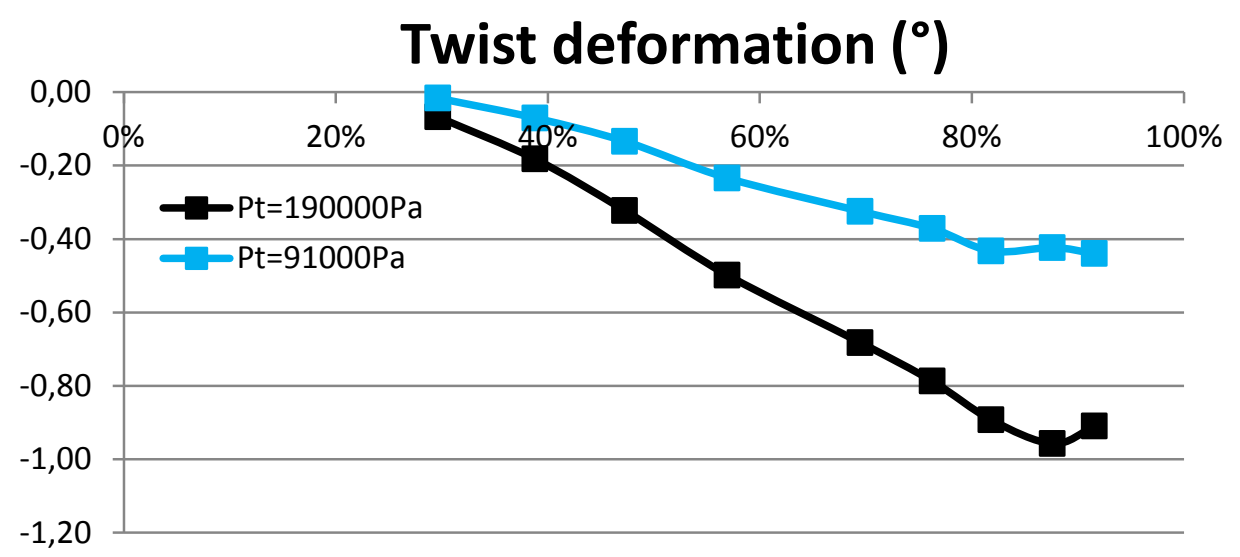

Fig. 14 Model Twist Deformation of LRM-S2MA model at $M a=0.85$ and Alpha=2. $7^{\circ}$.

The effects on loads are analyzed in the next section.

\section{E. Reynolds number and aeroelastic effects on model loads}

1. Numerical simulations details

The wing geometries used for the numerical simulations are the one proposed in the DPW-6. They are at scale 1/1, and a Reynolds number of 5 million based on MAC. The meshes "Overset grids Boeing Serrano.REV00" available on the DPW website [3] have been used, the medium grids have been used. In addition to the grids of the WB configuration presented above, the ONERA grids of the horizontal and vertical tails introduced in [11] have been used.

Before running these grids from Boeing with the elsA solver, a necessary pre-processing has been handled. Eight different bases describe the WB configuration: three external boxes, the fuselage body and fuselage nose, the collar grid at wing-body junction, the wing itself, and the wing tip. Along the whole process, the in-house software Cassiopee [16] has been extensively used. The blanking step which consists in removing all cells that are inside physical bodies has been realized using the latest Cassiopee blanking function. More detail can be found in [7].

As mentioned previously, all the computations have been performed with elsA [17].This software uses a cell-centered finite-volume discretization on structured point-matched or Overset grids. In this study, time integration is carried out by a backward-Euler scheme with implicit LU-SSOR relaxation. Spatial discretization is realized using a $2^{\text {nd }}$ order central Jameson scheme [18] with artificial viscosity. Multigrid techniques (one level) are used to accelerate convergence.

Turbulence effects have been simulated with the one-equation Spalart-Allmaras model [19] with the Quadratic Constitutive Relation (QCR) proposed in [20]; it leads to a nonlinear version of the model which does not use the traditional Boussinesq relation anymore.

The Overset interpolations are classically performed over two cell layers around holes and overlap conditions and a double-wall algorithm is used to ensure accurate interpolations when surfaces are described by several grids (collar grid for instance).

2. Reynolds number effect

Some CFD calculations on the pure Reynolds effect have been carried out on the model using the DPW-6 grids. The CFD results using scale $1 / 1$ geometries are added in purple, the Reynolds number effect being assessed on scale 1/50 geometries at test conditions (black and blue symbols). The effects on lift are presented in Fig. 15. At cruise point, the Reynolds number effect is about $-0.15^{\circ}$ of Alpha. The effects on pitching moment are very small and presented in Fig. 16. The effects on drag are presented in Fig. 18, showing a decrease of about 20 counts of drag between $\operatorname{Re}_{M A C}=3.6$ million to $\operatorname{Re}_{M A C}=1.8$ million. As expected, Fig. 18 shows that a great part comes from friction drag (17 d.c.).

It can be added that the use of the classical "Prandtl-Schlichting skin friction formula for a smooth flat plane at zero incidence" gives a good estimation of the Reynolds number effect on friction drag: about 18 d.c. from a Reynolds number of 3.6 million to a Reynolds number of 1.8 million.

$$
D C X_{f}=C X_{f}\left({\frac{\log _{10} R E}{\log _{10} R E_{C}}}^{2,58}-1\right)(4)
$$




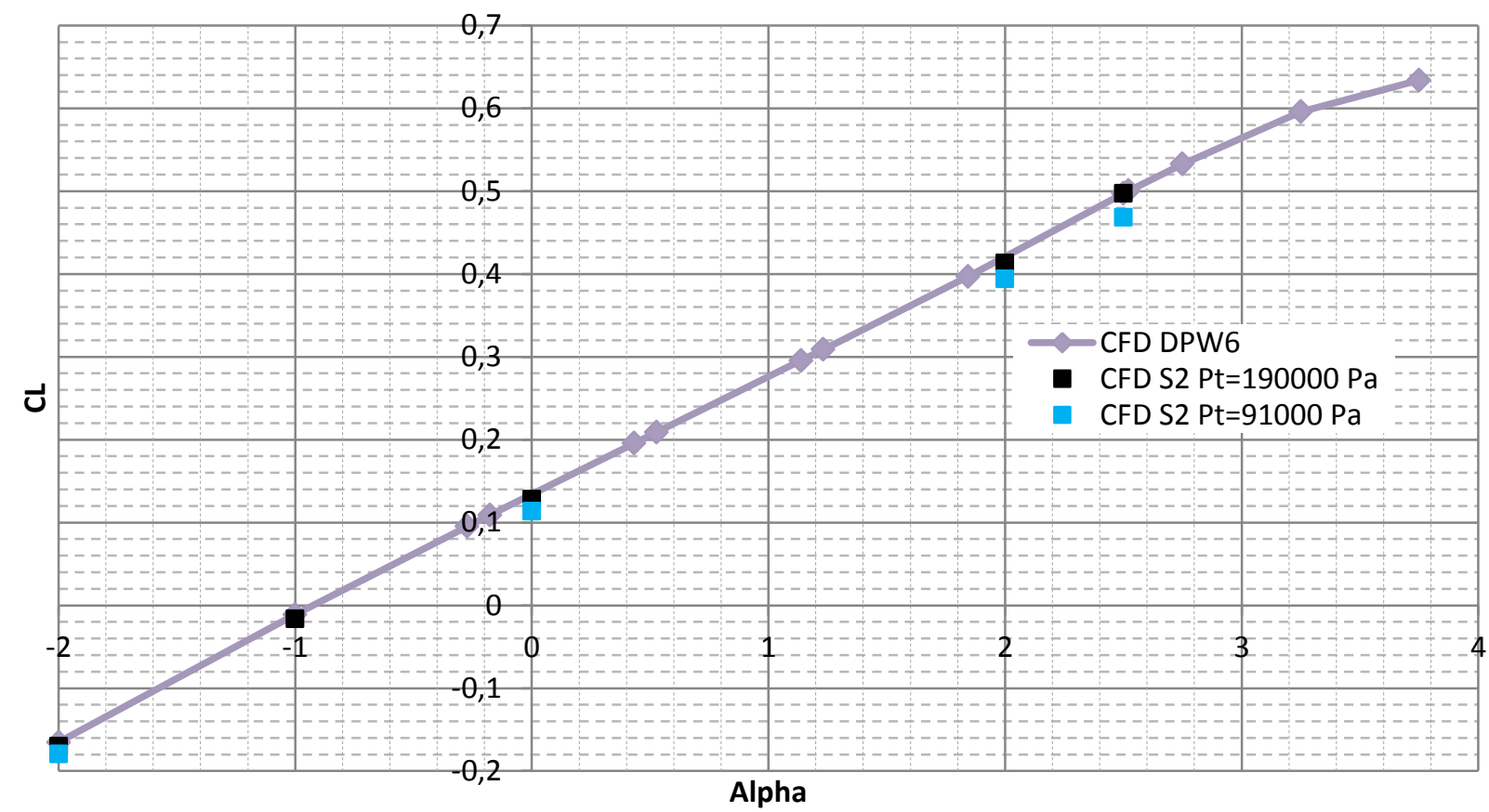

Fig. 15 Reynolds number effect on lift calculated by CFD at $M a=0.85$ on WBVH configuration.

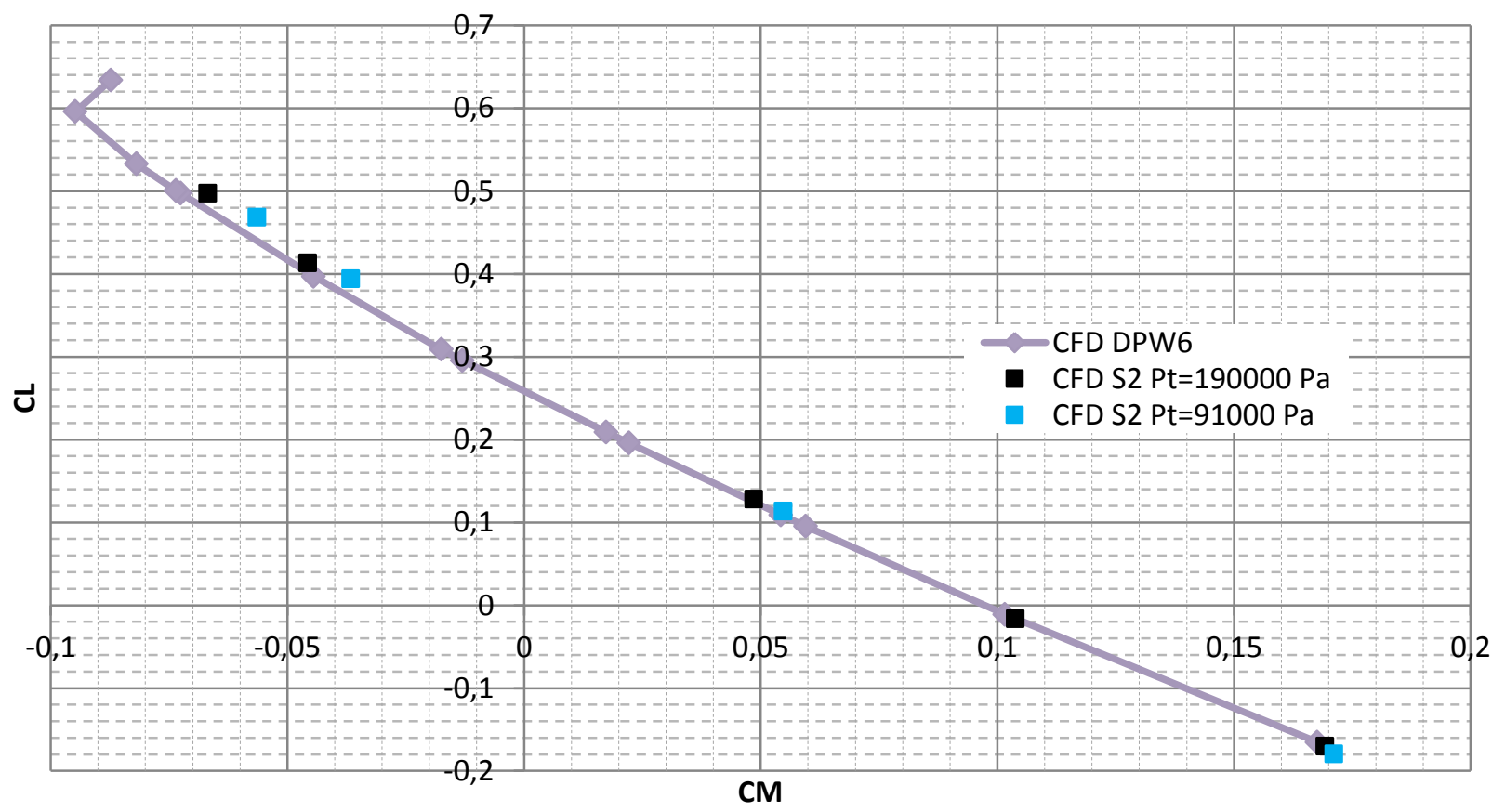

Fig. 16 Reynolds number effect on pitching moment calculated by CFD at $M a=0.85$ on WBVH configuration. 


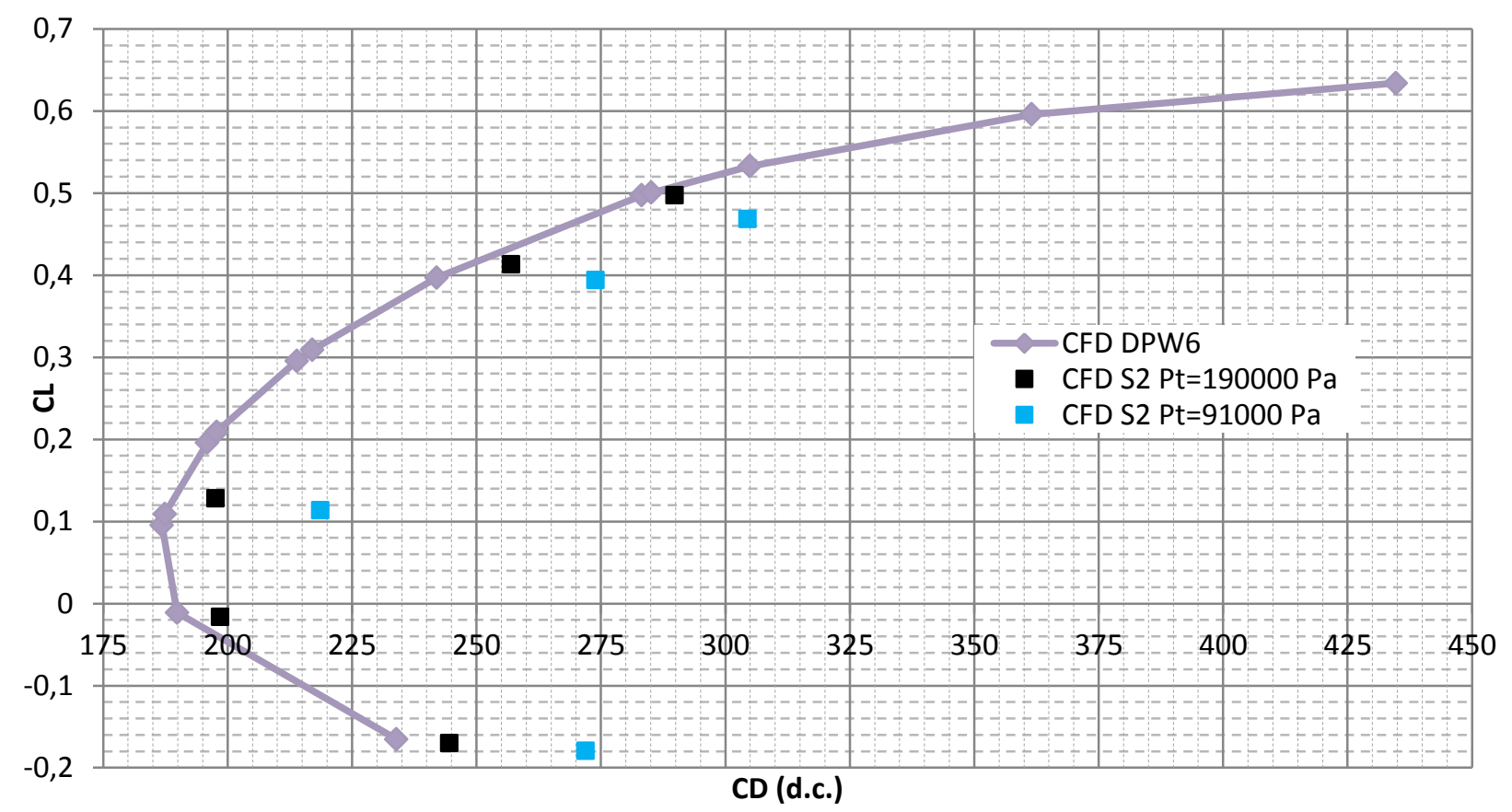

Fig. 17 Reynolds number effect on drag calculated by CFD at $M a=0.85$ on WBVH configuration.

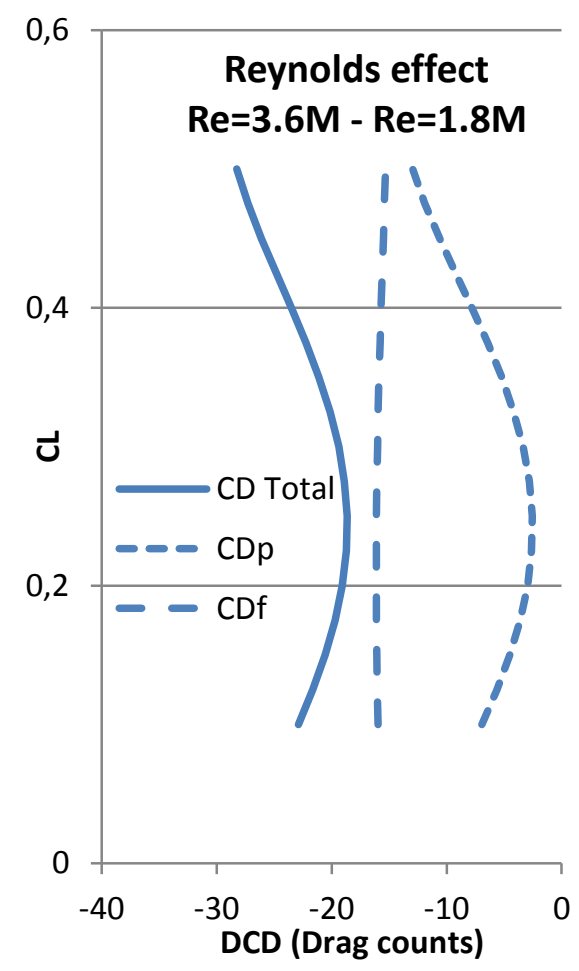

Fig. 18 Reynolds number effect on near field drag calculated by CFD at $M a=0.85$ on WBVH configuration. 


\section{Combined Reynolds and aeroelastic effect}

As said previously, a higher total pressure causes outer wing section to pitch nose down. The local lift coefficient of these sections diminishes, so the lift coefficient of the model should also decreases.

The experimental effects of deformation combined with Reynolds number changes are shown in Fig. 19. The results are presented in deltas, with the high Reynolds number in reference. It shows that the Reynolds number effect compensates the deformation effect, leading to very small changes on the lift coefficient (the image in the middle shows very small effect on lift).

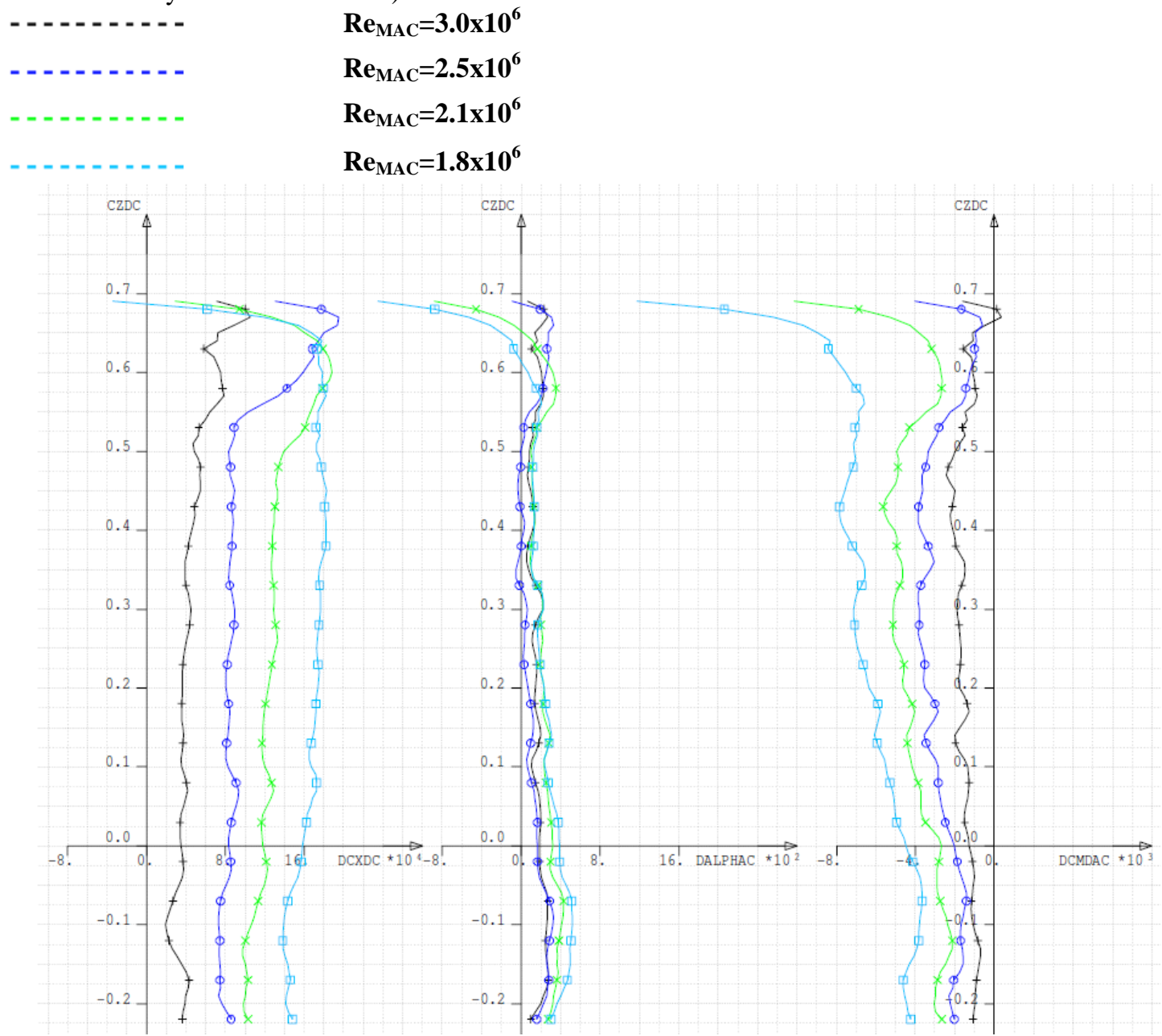

Fig. 19 Total pressure and Reynolds number effects measured at $M a=0.85$ on WBVH configuration, $\operatorname{Re}_{M A C}=3.6 \times 10^{6}$ as reference.

A higher total pressure is also expected to produce a higher pitching moment coefficient (outer wing located behind the reference center of the model), as it can be seen on the right image of Fig. 19 (negative delta for a lower total pressure). This is entirely caused by a deformation effect, the Reynolds number effect on pitching moment being very small as calculated by CFD (see Fig. 16). The effect of a model deformation change on the pitching moment coefficient was calculated by D. Hue (see Fig 15 of [6]): a change of $1^{\circ}$ of the model twist at wing tip induced a change of the pitching moment coefficient of $25.10^{-3}$. In our case, a change of $0.5^{\circ}$ of the model twist at wing tip induces a change of the pitching moment coefficient of $8.10^{-3}$ (light blue curve of Fig. 19 at $C L=0.5$ ).

The effect on drag is predictable and almost entirely due to a Reynolds number effect. Fig. 19 (left image) shows a decrease of about 17 drag counts (d.c.) when the total pressure is increased from $90000 \mathrm{~Pa}$ to $190000 \mathrm{~Pa}$ (and the Reynolds number as well).

The effect between the highest and the lowest Reynolds number is summarized in increments in Fig. 20 for $M a=0.85$. The CFD pure Reynolds number effect and the combined experimental Reynolds number and aeroelastic effects make it possible to guess the pure aeroelastic effects as depicted in Fig. 20 (green curve). This is consistent with what would be expected: a low effect on drag, a lower lift with a higher total pressure (so a greater angle of attack at same lift), a higher pitching moment with a higher total pressure, 

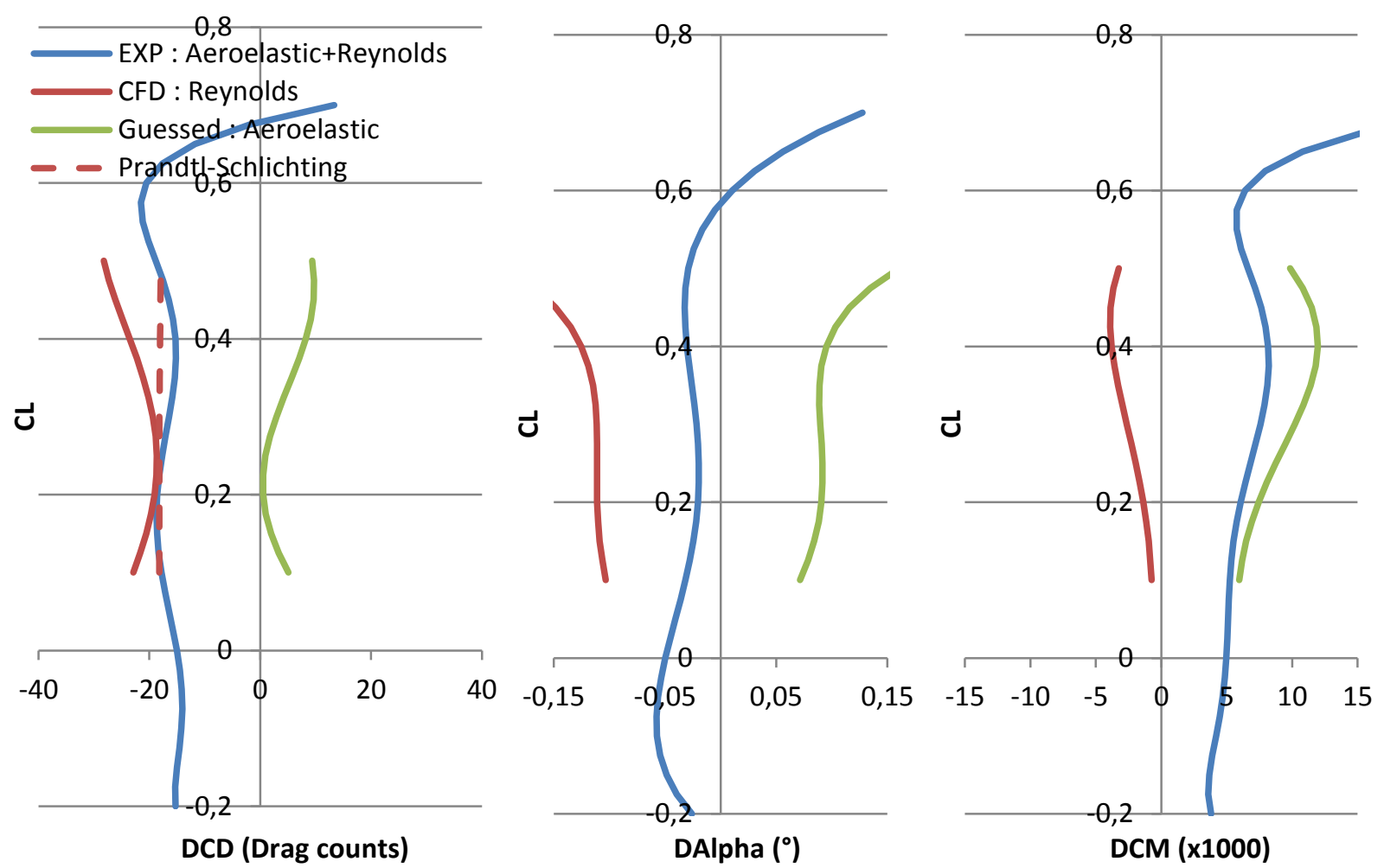

Fig. 20 Total pressure and Reynolds number effects at $M a=0.85$ on WBVH configuration $(\operatorname{Re}=3.6 \mathrm{M}-$ $\operatorname{Re}=1.8 M$ ).

\section{F. First comparison with S1MA results}

1. Wing pressure

The pressure distributions obtained on the WBVH configuration at Ma=0.85 and $C L=0.5$ are shown in Fig. 21. Four spanwise wing stations are in common between S1MA and S2MA test results: $\eta=0.201,0.502$ and 0.727 (on both wings).

The data compare well on the entire wing, at cruise wing. This was expected as the wing shape is the same between LRMS1 and LRMS2 at that point.

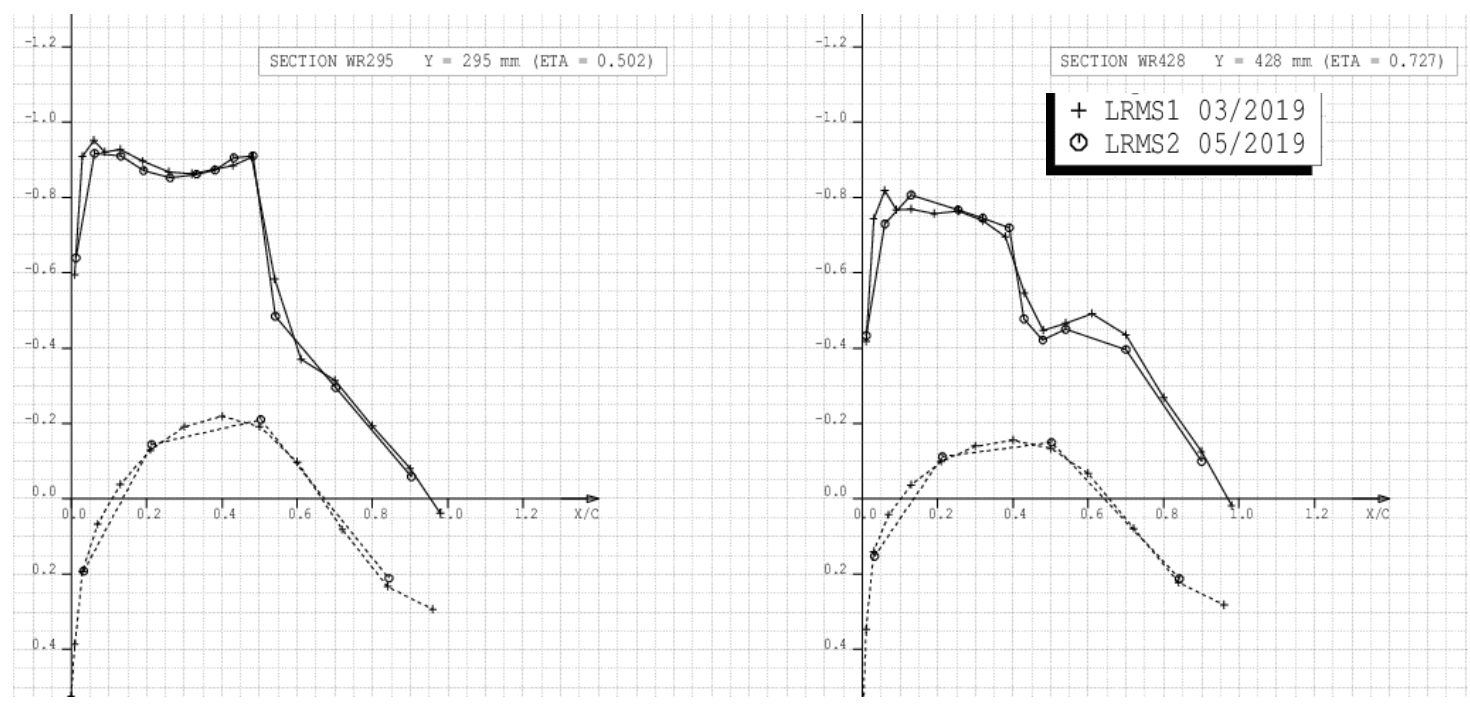




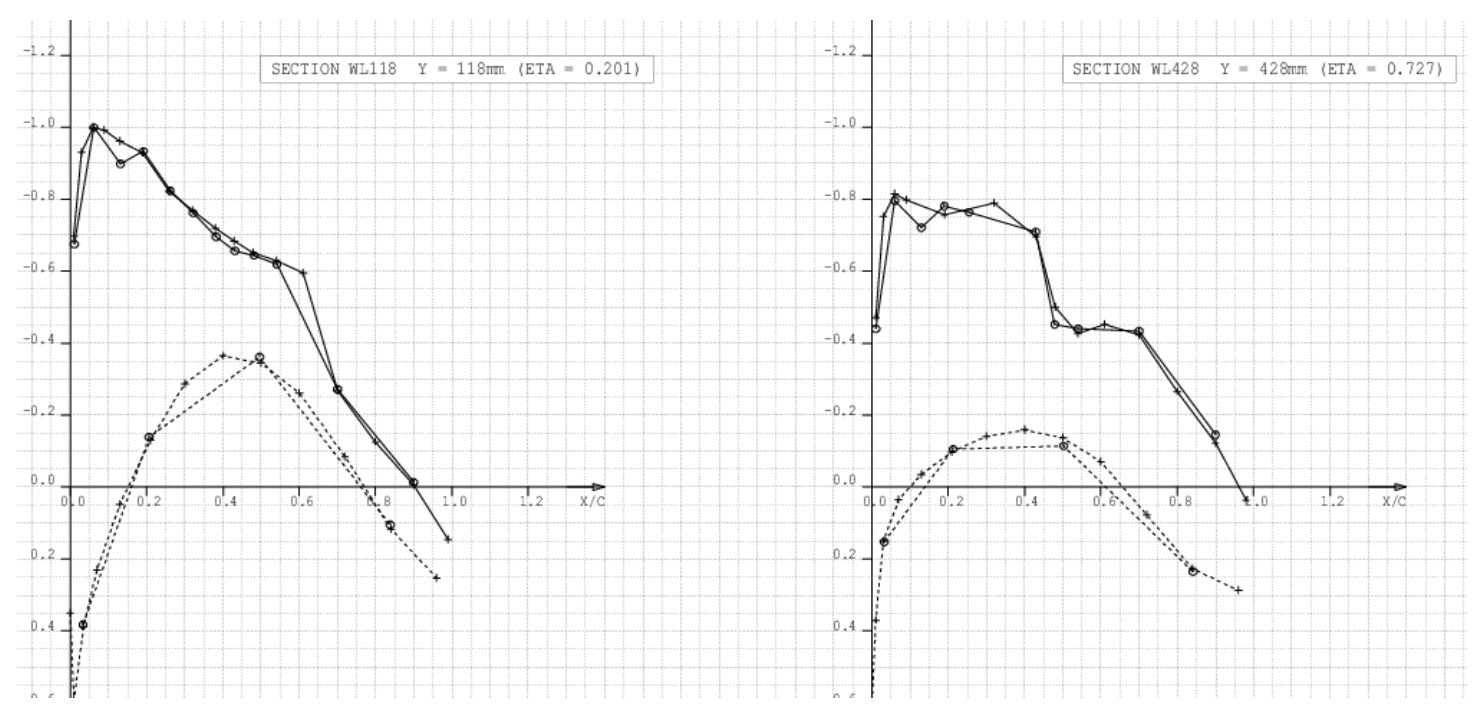

Fig. 21 Pressure distribution for $\mathrm{WBVH}$ at $M a=0.85$ and $C L=0.5$.

2. HTP pressure

Then, some comparisons on pressure distribution on the HTP can be made between S1MA and S2MA results. As shown in Fig. 22 the two set ups are different. The S2MA sting is located much closer to the HTP, compared to the S1MA set-up. The CRM set-up has been added in green.

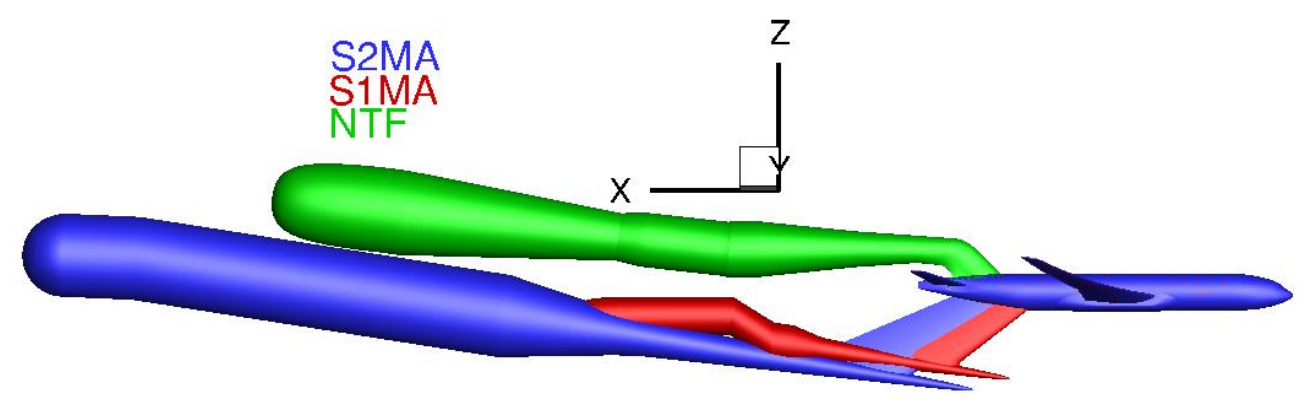

Fig. 22 Wind tunnel set up.

As mentioned in section II-D, the wind tunnel results (force and moment) have been corrected for support effects by means of CFD computations. Therefore, pressure coefficient disturbance caused by the support has been computed as depicted in Fig. 23. It is clearly visible that the S1MA sting mainly impacts the HTP lower side, whereas the S2MA sting impacts both upper and lower sides. As a result, the effective local incidence on the HTP is more strongly decreased (and the model nose-up pitching moment increased) in the S1MA case than in the S2MA case, which is visible on the HTP lifting surface.

It is then allowed to compare pressure distortion between S2MA and S1MA computed and measured (see Fig. 24, circles indicate pressure taps colored by experimental results). The results compare well, showing that the support effects calculated by CFD are consistent. 


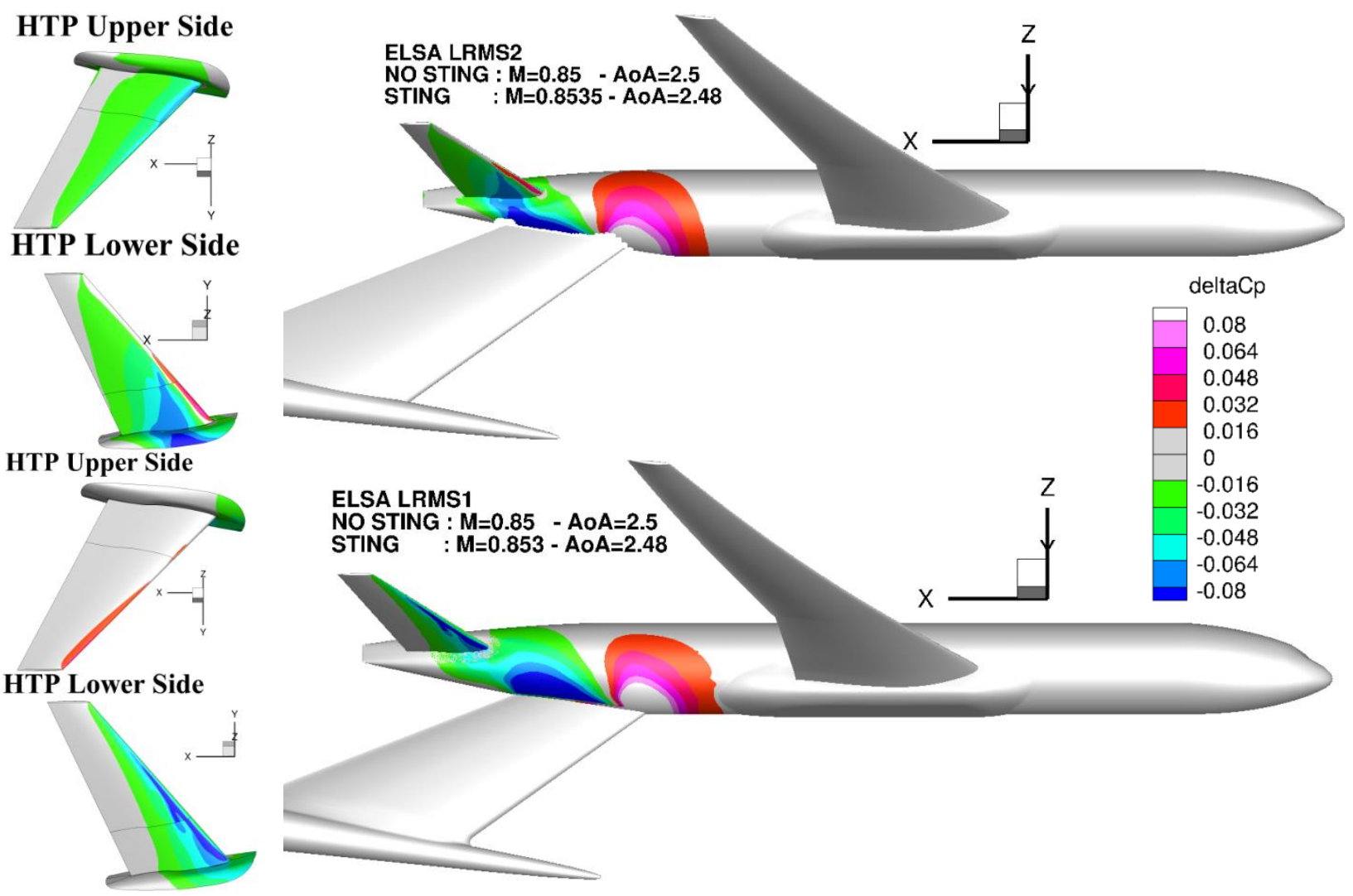

Fig. 23 Pressure coefficient disturbance on model skin (with sting minus without sting) at $M a=0.85$ and $C L=0.5$, S2MA top and S1MA bottom.
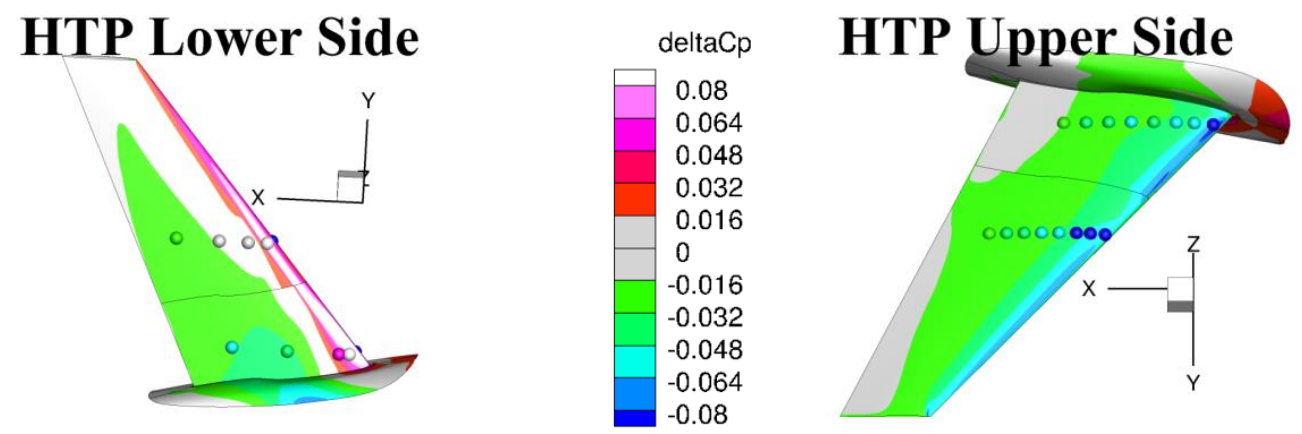

Fig. 24 Pressure coefficient distortion on model skin (S2MA minus S1MA) at $M a=0.85$ and $C L=0.5$.

\section{Loads}

The lift, drag and pitching moment results at cruise point are presented in Table 2. The drag is 20 d.c. higher in S2MA than in S1MA, and the pitching moment is 0.01 lower in S2MA than in S1MA.

At that point, the comparison should be perfect if:
1) the wing deformation is the same between S1MA and S2MA;
2) the Reynolds number is the same between S1MA and S2MA;
3) the wall and support corrections are well taken care of in both facilities;
4) the surface roughness of the model is low enough;
5) the model surface definition is the same between LRM-S1MA and LRM-S2MA.

Point 1 has been checked and isn't expected to be a source of discrepancy. Point 2 is small enough not to induce too much difference. Indeed, S2MA Reynolds number is 3.6 million and S1MA is 4.8 million so not more than 8 drag counts is expected. Point 3 has been carefully computed, both in S1MA [21] and S2MA. Point 4 is likely to have an important effect on the results. The surface roughness is about the same order of magnitude for LRM-S1MA and LRM-S2MA, but the scale difference (1/16.835 for S1MA and 1/50 for S2MA) leads to a higher Reynolds number per meter at cruise point at S2MA. Therefore, it is expected to have a higher roughness 
effect at S2MA than at S1MA. This effect is under evaluation to access its impact on the model coefficients. Point 5 has been checked during the model inspection.

Table 2 Balance measurements, comparison between S1MA and S2MA at $M a=0.85$ and $C L=0.5$ on WBVH configuration.

\begin{tabular}{|c|c|}
\hline & S2MA-S1MA \\
\hline dAlpha & $\mathbf{0 . 0 6}^{\circ}$ \\
\hline$d C D($ in d.c. $)$ & 20 \\
\hline$d C M\left(\mathrm{x} 10^{3}\right)$ & -10 \\
\hline
\end{tabular}

\section{Conclusions and perspectives}

The new ONERA S2MA Large Reference Model has been tested for the first time in the S2MA wind tunnel. Data have been obtained at different Reynolds numbers, for the WBVH configuration. Force and moment, surface pressure and infrared visualizations were obtained over a large number of test conditions.

First, some natural laminar visualisations were conducted showing the Reynolds number effect.

Then, the effectiveness of the boundary layer tripping was checked with fixed transition infrared visualizations.

The model deformation measurements were conducted, confirming that the wing had the same shape between S1MA and S2MA (for a high total pressure) at cruise point.

Some Reynolds number (along with dynamic pressure effects) were carried out and partly explained. CFD investigations on model deformation effect are under study in order to confirm the measured combined effects.

Finally, some comparison between S1MA and S2MA results were performed.

1) Pressure

The data compare well on the wing. On the HTP, CFD support effects are in good agreement with the experimental pressure distribution distortion between S1MA and S2MA;

2) Forces and moment

At cruise point, where the results should give the better comparison because of the model deformation, some discrepancies remain.

The first step toward a wind tunnel comparison is to test the same model with the same sting in S1MA and S2MA. This is possible using the LRM-S2MA model and will be carried out shortly, using the same dynamic pressure (same Reynolds number and dynamic pressure). The S1MA results would serve as a reference, and validate the S2MA results. The LRM-S2MA model will then be tested in S1MA wind-tunnel as soon as possible.

Then, configuration effects are planned to be tested in S2MA, so that the pitching moment difference between LRM-S1MA and LRM-S2MA can be better understood.

CFD investigations on the model roughness effect on wind tunnel results are in progress and will be presented in coming articles.

Outside cruise point, CFD investigations on the model deformation effect between S1MA and S2MA results are in progress allowing comparing and explaining the results obtained in the two wind tunnels (that lead to different model deformation).

At last, ONERA is currently working on high lift version of the CRM, with the same geometry as the CRMHL. 


\section{References}

${ }^{1 .}$ Vassberg, J. C., DeHann, M. A., Rivers, S. M., and Wahls, R. A., "Development of a Common Research Model for Applied CFD Validation Studies," AIAA Paper 2008-6919, 2008.

2.NASA, Langley Research Center, “Common Research Model,” http://commonresearchmodel.larc.nasa.gov/.

${ }^{3}$ DPW-6 website, http://aiaa-dpw.larc.nasa.gov/

4.Hue, D., and Esquieu, S., "Computational Drag Prediction of the DPW4 Configuration Using the Far-Field Approach," Journal of Aircraft, Vol. 48, No. 5, Sept.-Oct. 2011, pp. 1658-1670.

${ }^{5 . H u e, ~ D ., ~ " F i f t h ~ D r a g ~ P r e d i c t i o n ~ W o r k s h o p: ~ C o m p u t a t i o n a l ~ F l u i d ~ D y n a m i c s ~ S t u d i e s ~ C a r r i e d ~ O u t ~ a t ~ O N E R A, " ~ J o u r n a l ~ o f ~}$ Aircraft, Vol. 51, No. 4, July.-August. 2014, pp. 1295-1310.

${ }^{6 .}$ Hue, D., "Fifth Drag Prediction Workshop: ONERA Investigations with Experimental Wing Twist and Laminarity," Journal of Aircraft, Vol. 51, No. 4, July.-August. 2014, pp. 1311-1322.

${ }^{7}$ Hue, D., Chanzy, Q., and Landier, S., "DPW-6: Drag Analyses and Increments Using Different Geometries of the CRM Airliner," Journal of Aircraft Vol. 55, No. 4, July.-August. 2018, pp. 1491-1310.

${ }^{8 .}$ Cartieri, A., Hue, D., Chanzy, Q., and Atinault, O., "Experimental Investigations on Common Research Model at ONERA-S1MA - Drag Prediction Workshop Numerical Results," Journal of Aircraft Vol. 55, No. 4, July.-August. 2018.

${ }^{9}$ ONERA S1MA website, http://windtunnel.onera.fr/s1ma-continuous-flow-wind-tunnel-atmospheric-mach-005-mach-1

${ }^{10}$ ONERA S2MA website, https://www.onera.fr/en/windtunnel/s2ma-continuous-flow-wind-tunnel-variable-pressuremach-01-mach-31

${ }^{11}$ Hue, D., et al., "Validation of a near-body and off-body grid partitioning methodology for aircraft aerodynamic performance prediction," Computers \& Fluids, Vol. 117, 2015, pp. 196-211.

${ }^{12 .}$ Le Sant, Y., Mignosi, A., Touron, G., Deléglise, B., Bourguignon, G., "Model Deformation Measurement (MDM) at ONERA", 25th AIAA Applied Aerodynamics Conference, AIAA 2007-3817, Miami, 25-28, June 2007

13. Vaucheret X., "Recent Calculation Progress on Wall Interferences in Industrial Wind Tunnels", La Recherche Aérospatiale, No. 3, pp 45-47,1988.

${ }^{14}$ Pistolesi, E., "Collected lectures of the principal meeting of the Lilienthal society", Considering respecting the mutual influence of system of airfoils, Berlin, 1937.

15. Cartieri, A., and Mouton, S., "Using CFD to calculate support interference effects," AIAA Paper 2012-2864, 2012.

${ }^{16}$ Péron, S., Benoit, C., Landier, S., and Raud, P., "Cassiopée: CFD Advanced Set of Services In an Open Python EnvironmEnt," 12th Symposium on Overset Grid and Solution Technology, Atlanta, 2014.

${ }^{17 .}$ Cambier, L., Heib, S., and Plot, S., "The ONERA elsA CFD Software: Input from Research and Feedback from Industry," Mechanics and Industry, Vol. 15(3), pp. 159-174, 2013.

18. Jameson, A., Schmidt, W., and Turkel, E., "Numerical Solution of the Euler Equations by Finite Volume Methods Using Runge Kutta Time Stepping Schemes,” AIAA-81-1259, June 1981.

${ }^{19 .}$ Spalart, P. R., and Allmaras, S. R., "A One-Equation Turbulence Model for Aerodynamic Flows," AIAA Paper 92-0439, 1992.

${ }^{20 .}$ Spalart, P. R., "Strategies for Turbulence Modelling and Simulation," International Journal of Heat and Fluid Flow, Vol. 21, 2000, pp. 252-263.

${ }^{21 .}$ Cartieri, A., and Hue, D., "Using RANS computations to calculate support interference effects on the Common Research Model," Advanced Wind Tunnel Boundary Simulation Research Workshop AVT-284 Paper STO-MP-AVT-284, 2018. 